\title{
Eulerian Gaussian Beams for Schrödinger Equations in the Semi-Classical Regime
}

\author{
Shingyu Leung* Jianliang Qian ${ }^{\dagger}$
}

August 21, 2008

\begin{abstract}
We propose Gaussian-beam based Eulerian methods to compute semi-classical solutions of the Schrödinger equation. Traditional Gaussian beam type methods for the Schrödinger equation are based on the Lagrangian ray tracing. We develop a new Eulerian framework which uses global Cartesian coordinates, level-set based implicit representation and Liouville equations. The resulting method gives uniformly distributed phases and amplitudes in phase space simultaneously. To obtain semi-classical solutions to the Schrödinger equation with different initial wave functions, we only need to slightly modify the summation formula. This yields a very efficient method for computing semi-classical solutions to the Schrödinger equation. For instance, the proposed algorithm requires only $O\left(s N n^{2}\right)$ operations to compute $s$ different solutions with $s$ different initial wave functions under the influence of the same potential, where $N=O(1 / \hbar)$, $\hbar$ is the Planck constant, and $n \ll N$ is the number of computed beams. Numerical experiments indicate that this Eulerian Gaussian beam approach yields accurate semi-classical solutions even at caustics.
\end{abstract}

\section{Introduction}

It is well known that classical mechanics describes the behavior of macroscopic objects, while quantum mechanics describes the behavior of microscopic objects with physical characteristics comparable in values with the Planck constant, such as elementary particles, atoms, and molecules. For macroscopic objects the Planck constant is considered to be negligible so that quantum effects can be ignored and classical mechanics in this regime provides a satisfactory approximation. However, for microscopic objects it is critical to incorporate quantum effects into modeling for the best physical fidelity. There are several equivalent quantum mechanics models, such as Schrödinger's scheme, Heisenberg's scheme, and Feyman's path-integral scheme.

Here we consider the Schrödinger equation for a particle with unity mass

$$
\begin{aligned}
& -i \hbar U_{t}+V(x) U-\frac{\hbar^{2}}{2} \Delta U=0, \quad x \in R^{n}, \quad t>0, \\
& U(x, 0)=A_{0}(x) \exp \left(\frac{i \tilde{\tau}_{0}(x)}{\hbar}\right)
\end{aligned}
$$

\footnotetext{
${ }^{*}$ Department of Mathematics, UCI, Irvine, CA 92697-3875. Email: syleung@math.uci.edu

${ }^{\dagger}$ Department of Mathematics, Michigan State University, East Lansing, MI 48824. Email: qian@math.msu.edu
} 
where $V$ is real and smooth, $\tilde{\tau}_{0}$ and $A_{0}$ are smooth, and $\hbar \equiv h / 2 \pi$ with $h$ a small (scaled) Planck's constant. This equation is obtained by introducing undulatory theory into classical mechanics, the so-called quantization procedure. Because the Schrödinger equation propagates oscillations of wavelength $\hbar$ in space and time, resolving such oscillations by direct numerical simulation requires many grid points per wavelength of $O(\hbar)$, which is costly in practice. As an alternative to obtain numerical approximation capturing quantum effects, semi-classical methods are sought to link classical and quantum mechanics. In this paper, we design an Eulerian Gaussian-beam method for the Schrödinger equation in the semi-classical regime, where the Planck constant is small.

The spirit of semi-classical approximation is expanding the quantum wave function around classical expressions in powers of contributions from quantum fluctuations. In the simplest case, we consider the following leading-order WKBJ ansatz for the quantum wave function,

$$
U(x, t) \approx A(x, t) \exp \left(\frac{i \tau(x, t)}{\hbar}\right)
$$

where $A(x, t)$ is the amplitude function and $\tau(x, t)$ is the phase function. Applying this ansatz to the Schrödinger equation and considering the leading order singularities, we have the following eikonal equation for the phase function and the transport equation for the amplitude function,

$$
\begin{aligned}
& \tau_{t}+V(x)+\frac{1}{2} \tau_{x}^{2}=0, \\
& A_{t}+\tau_{x} \cdot A_{x}+\frac{1}{2} \Delta \tau A=0
\end{aligned}
$$

with the initial data,

$$
\begin{aligned}
\tau(x, 0) & =\tilde{\tau}_{0}(x) \\
A(x, 0) & =A_{0}(x) .
\end{aligned}
$$

The transport equation for the amplitude is weakly coupled to the eikonal equation in the sense that one must first solve the eikonal equation to provide related coefficients for the transport equation. Because the eikonal equation is a non-linear first-order equation, in general there exists no global smooth (classical) solutions for the equation. Consequently, the WKBJ ansatz (3) might not be valid globally in terms of global smooth eikonal and amplitude. One remedy is to seek globally defined unique weak solutions in physical space to the eikonal equation, which leads to the so-called viscosity solution [8]. Another remedy is to solve the eikonal equation in phase space first using the method of characteristics and then projecting the resulting solution into physical space. The essential difference between these two remedies can be seen right away: the viscositysolution based eikonal solution in physical space results in a single-valued phase function while the method-of-characteristics based eikonal solution in physical space might result in a multivalued phase function. Which remedy is more appropriate depends on specific applications.

For linear Schrödinger equations, multivalued phases are appropriate, and the resulting amplitudes are also multivalued. As a result, the WKBJ ansatz (3) should be modified to accommodate multivalued phases and amplitudes, which has been done systematically in terms of uniform asymptotic solutions in $[19,24]$. In both [19] and [24] one has to pay particular attention to so-called caustics, where the projections of phase-space bi-characteristics into physical space, the so-called 
rays, converge and the resulting amplitudes become infinite. In [19] one has to identify caustics so as to choose appropriate expanding functions, such as Airy or Pearcy functions, to treat the boundary layer effect near caustics. In [24] one has to keep track of the so-called Keller-Maslov index to compensate for the phase shift when passing through a caustic. In general, since caustics can occur anywhere along the ray, the above two approaches are not satisfactory. In contrast, Gaussian beams provide a powerful framework for constructing uniform asymptotic solutions systematically even at caustics without identifying caustics or keeping track of Keller-Maslov index.

The idea underlying Gaussian beams is simply to build asymptotic solutions to partial differential equations concentrated on a single curve through the domain; this single curve is nothing but a ray as shown in [32]. The existence of such solutions has been known to the pure mathematics community since sometime in the 1960s [1], and these solutions have been used to obtain results on propagation of singularities in hyperbolic PDEs [13, 32]. An integral superposition of these solutions can be used to define a more general solution that is not necessarily concentrated on a single curve. Gaussian beams can be used to treat pseudo-differential equations in a natural way, including Helmholtz and Schrödinger equations.

In the geophysical applications, Gaussian beam superpositions have been used for seismic wave modeling [6] and for seismic wave migration [12]. The numerical implementations in these works are based on ray-centered coordinates which prove to be computationally inefficient. More recently, based on $[32,36]$ a purely Eulerian computational approach was proposed in [17] which overcomes some of these difficulties. In [36] Gaussian beams are successfully constructed to simulate mountain waves, a kind of stationary gravity waves forming over mountain peaks and interfering with aviation.

In quantum mechanics, some variants of Gaussian beams, such as frozen Gaussian beams and Gaussian wave packets, have been used to construct approximate solutions to Schrödinger equations in the semi-classical regime $[16,10,11]$. However, these formulations were all based on the Lagrangian framework. In this paper, we propose an Eulerian formulation for Gaussian beams. We first follow the ansatz proposed in $[32,36]$ to construct Gaussian beams to the Schrödinger equation along central rays. Mathematically, this ansatz constructs an approximate phase function with an imaginary part as a Taylor expansion around a central ray by using phase derivatives on the central ray. To have a corresponding Eulerian formulation capturing multi-valued phases and caustics, we generalize an Eulerian Gaussian beam approach proposed in [17] to the Schrödinger equation. The Eulerian method proposed in [17] is based on level sets and paraxial Liouville equations [29, 18, 30] and is designed to solve Helmholtz equations in the high frequency regime.

The advantages of this Eulerian Gaussian Beams approach over the usual Lagrangian framework are multi-folded. Unlike usual Lagrangian formulations, we obtain a uniform resolution of ray distribution so that the resulting Gaussian beam summation will have a uniform resolution as well. To obtain another asymptotic solution to the Schrödinger equation with a different initial wave function or for a different parameter $\hbar$, we only slightly modify the summation formula. This results in a computationally very efficient algorithm when we are solving the Schrödinger equation especially for various settings of the initial wave function under the same potential. For instance, the proposed algorithm requires at most $O\left(s N n^{2}\right)$ operations to compute $s$ different solutions with $s$ different initial wave functions under the same potential, where $N=O(1 / \hbar)$, and $n \ll N$ is the number of beams we apply.

We remark that there are some other recent works for solving the Schrödinger equation in the semi-classical regime. In [21] the authors apply Wigner-transform techniques to the analysis of finite 
difference methods for the Schrödinger equation in the case of a small Planck constant; in terms of numerical approximations of quadratic observables rather than the quantum wave function itself, they are able to obtain Wigner-measure related sharp conditions on the spatial-temporal grid which guarantee convergence for average values of observables as the Planck constant tends to zero. In [2] the authors propose time-splitting spectral approximations for the linear Schrödinger equation in the semi-classical regime; in terms of numerical approximations of quadratic observables rather than the quantum wave function itself, their numerical examples and analytical considerations based on the Wigner transform show that weaker constraints on mesh sizes for the direct numerical solution of the Schrödinger equation are admissible for obtaining "correct" observables. In terms of computing multivalued phases and amplitudes in an Eulerian framework, an approach based on level sets and Liouville equations first initiated in [27] and further developed in [28, 7, 15], etc, has been successful; as a further development along this line, in [14] an Eulerian approach is proposed for computing multivalued physical observables for the Schrödinger equation in the semi-classical regime, but the approach unavoidably runs into difficulty at caustics, and it does not construct the quantum wave function either. Unlike $[21,2]$ where physical observables are computed from numerical solutions obtained by direct numerical methods for the Schrödinger equations, the Eulerian Gaussian beam approach proposed here computes the observables from uniform asymptotic solutions of the Schrödinger equation. Since the beam-based asymptotic solution is valid even at caustics, our Eulerian Gaussian beam approach is different from the approach in [14].

The rest of the paper is organized as follows. In Section 2, we summarize the Lagrangian Gaussian beam formulation and develop four different methods for initializing beam propagation. In Section 3, we develop an Eulerian Gaussian beam formulation and develop a semi-Lagrangian method for solving the resulting level-set equations and Liouville equations. In Section 4, we analyze the complexities of the resulting algorithms. In Section 5, we show numerical examples to demonstrate the efficiency and accuracy of the algorithms.

\section{Lagrangian Gaussian Beams (LGB)}

\subsection{Construction of Gaussian beams}

We would like to construct an asymptotically valid solution $\Psi(x, t ; \hbar)$ for the Schrödinger equation, that is concentrated on a single curve $\gamma$. Namely, $|\Psi(x, t ; \hbar)|$ is small away from $\gamma$, and it will satisfy the Schrödinger equation up to $O\left(\hbar^{M}\right)$ for some fixed positive number $M$ under some appropriate norm. Here we are interested in constructing the lowest-order Gaussian beam so that the Schrödinger equation will be satisfied up to $O(\sqrt{\hbar})$

To construct such Gaussian beams for the Schrödinger equation,

$$
-i \hbar U_{t}+V(x) U-\frac{\hbar^{2}}{2} \Delta U=0, \quad x \in R^{n}, \quad t>0,
$$

we follow $[32,36,23]$ and start with the WKBJ ansatz,

$$
U(x, t) \approx A(x, t) \exp \left(\frac{i \tau(x, t)}{\hbar}\right) .
$$

The functions $A(x, t)$ and $\tau(x, t)$ are all assumed to be smooth, and these requirements are feasible because the beam solution is constructed to be concentrated on a single curve; this is the essential 
difference between traditional WKBJ asymptotic solutions and Gaussian beam solutions. As a result, the requirements on the phase function $\tau$ are slightly different from those of traditional WKBJ asymptotics. We will require that $\tau$ is real valued on $\gamma$, but away from this curve $\gamma, \tau$ can be complex valued with the restriction that the imaginary part of the second-order derivative $\tau_{x x}$ is positive definite. This will make $U$ look like a Gaussian distribution with variance $\hbar$ on planes perpendicular to $\gamma$.

Define the following Hamiltonian for the Schrödinger equation,

$$
H(x, p)=\frac{p^{2}}{2}+V(x) \text {. }
$$

According to the Gaussian beam theory [32], such a curve $\gamma$ is nothing but the $x$-projection of bicharacteristics $(x(t), p(t))$ satisfying the following Hamiltonian system,

$$
\begin{aligned}
& \dot{x}=\frac{d x}{d t}=H_{p},\left.\quad x\right|_{t=0}=x_{0}, \\
& \dot{p}=\frac{d p}{d t}=-H_{x},\left.\quad p\right|_{t=0}=p_{0},
\end{aligned}
$$

where $t$ is time parametrizing bicharacteristics. Along bicharacteristics, the phase function satisfies

$$
\dot{\tau}=\frac{d \tau}{d t}=\frac{1}{2} p^{2}-V(x),\left.\quad \tau\right|_{t=0}=\tau_{0}
$$

To determine the second order derivative $\tau_{x x}$ along bicharacteristics, we solve the following variational system for matrix-valued solutions $B(t)$ and $C(t)$ :

$$
\begin{aligned}
& \dot{B}=-H_{x p}^{T} B-H_{x x} C,\left.\quad B\right|_{t=0}=B_{0}, \\
& \dot{C}=H_{p p} B+H_{p x} C,\left.\quad C\right|_{t=0}=I,
\end{aligned}
$$

where $I$ is the identity matrix, and matrix $B_{0}$ is chosen to take into account the initial phase function and to have an imaginary part which is positive definite. Here $B=B\left(t ; x_{0}, p_{0}\right)$ and $C=C\left(t ; x_{0}, p_{0}\right)$ are taken to be the variations of $p=p\left(t ; x_{0}, p_{0}\right)$ and $x=x\left(t ; x_{0}, p_{0}\right)$ along the bi-characteristics with respect to the initial point $x_{0}=\alpha$,

$$
B\left(t ; x_{0}, p_{0}\right)=\frac{\partial p}{\partial \alpha}, \quad C\left(t ; x_{0}, p_{0}\right)=\frac{\partial x}{\partial \alpha} .
$$

We notice that $B C^{-1}$ yields the Hessian of the phase function $\tau$ along the bi-characteristics. Solution to the above equations exists on any interval $t \in[0, T]$. Moreover, we have the following lemma on the bound of the solution; its proof can be found in $[32,36]$.

Lemma 2.1. Under the above assumptions, $C(t)$ is non-singular for any $t$, and $\operatorname{Im}\left(B C^{-1}\right)$ is positive definite.

Since $p(t)=\tau_{x}(t)$ along bicharacteristics, we can use the following second-order Taylor expansion to define a smooth global approximate phase function:

$$
\begin{aligned}
\tau\left(x, t ; x_{0}, p_{0}\right)= & \tau\left(t ; x_{0}, p_{0}\right)+p\left(t ; x_{0}, p_{0}\right) \cdot\left(x-x\left(t ; x_{0}, p_{0}\right)\right) \\
& +\frac{1}{2}\left(x-x\left(t ; x_{0}, p_{0}\right)\right)^{T}\left(B C^{-1}\right)\left(x-x\left(t ; x_{0}, p_{0}\right)\right) .
\end{aligned}
$$


Next we need to determine the amplitude function $A$. According to the beam theory, the amplitude function $A$ satisfies the transport equation,

$$
\dot{A}=-\frac{1}{2} \operatorname{trace}\left(B C^{-1}\right) A,\left.\quad A\right|_{t=0}=A_{0}\left(x_{0}, p_{0}\right) .
$$

The initial condition $A_{0}$ depends on how the initial wave function is decomposed into a summation of Gaussians centered at different locations, as we will see in Section 2.2. Solution to this equation can be found analytically and is given by the following lemma.

Lemma 2.2. The solution for the transport equation (16) is

$$
A\left(t ; x_{0}, p_{0}\right)=\frac{A_{0}\left(x_{0}, p_{0}\right)}{\sqrt{\operatorname{det}\left(C\left(t ; x_{0}, p_{0}\right)\right)}} .
$$

Lemma 2.2 can be proved by applying the divergence theorem to equation (5) around a ray tube; this ray tube in the time-space domain is defined by intersecting two neighboring rays with two horizontal surfaces corresponding to $t^{\prime}=t_{1}$ and $t^{\prime}=t_{2}$; see [17] for a proof of a similar lemma.

To obtain a smooth global approximate amplitude function, we use the following extension:

$$
A\left(x, t ; x_{0}, p_{0}\right)=A\left(t ; x_{0}, p_{0}\right) .
$$

Inserting (15) and (18) into the WKBJ ansatz yields an asymptotically valid solution:

$$
\Psi\left(x, t ; x_{0}, p_{0}\right)=A\left(x, t ; x_{0}, p_{0}\right) \exp \left[i \frac{\tau\left(x, t ; x_{0}, p_{0}\right)}{\hbar}\right]
$$

this beam solution is concentrated on a single smooth curve $\gamma$ which is the $x$-projection of the bicharacteristic emanating from $\left(x_{0}, p_{0}\right)$ at $t=t_{0}=0$.

The asymptotic solution is obtained by integrating all the beams parametrized by the initial point $\left(x_{0}, p_{0}\right)$,

$$
U(x, t)=c(\epsilon, \hbar, n) \int_{p_{0}} \int_{x_{0}} \Psi\left(x, t ; x_{0}, p_{0}\right) d x_{0} d p_{0},
$$

for some normalization constant $c(\epsilon, \hbar, n)$.

\subsection{Initializing Gaussian beams}

We will consider the following highly oscillatory initial data for the Schrödinger equation:

$$
U(x, 0 ; \hbar)=U_{0}(x ; \hbar)=A_{0}(x) \exp \left(\frac{i \tilde{\tau}_{0}(x)}{\hbar}\right)
$$

where $A_{0}(x)$ and $\tilde{\tau}_{0}(x)$ are smooth functions, and $A_{0}(x)$ has compact support.

In practice, we may only know that $U_{0}(x ; \hbar)$ is highly oscillatory, but we may not know the specific expressions of $A_{0}(x)$ and $\tilde{\tau}_{0}(x)$. Therefore, we will initialize beam propagation by using different strategies and superpose them accordingly. 
Eulerian Gaussian Beams for Schrödinger Equations in the Semi-Classical Regime

\subsubsection{Initializing by asymptotic decomposition (AD)}

If $A_{0}(x)$ and $\tilde{\tau}_{0}(x)$ are given in specific expressions, then we may use the following strategy to initialize beam propagation:

$$
\begin{aligned}
\left.x\right|_{t=0} & =x_{0} \\
\left.p\right|_{t=0} & =p_{0}=\frac{\partial \tilde{\tau_{0}}}{\partial x}\left(x_{0}\right) \\
\left.\tau\right|_{t=0} & =\tilde{\tau}\left(x_{0}\right) \\
\left.B\right|_{t=0} & =B_{0}=\frac{\partial^{2} \tilde{\tau_{0}}}{\partial x^{2}}\left(x_{0}\right)+i \epsilon I \\
\left.C\right|_{t=0} & =I \\
\left.A\right|_{t=0} & =A_{0}\left(x_{0}\right)
\end{aligned}
$$

Consequently, the resulting beam ingredients are functions of $x_{0}$ only, and the approximate functions $\tau\left(x, t ; x_{0}, p_{0}\right)=\tau\left(x, t ; x_{0}\right)$ and $A\left(x, t ; x_{0}, p_{0}\right)=A\left(x, t ; x_{0}\right)$. Furthermore, the beam summation formula will be modified to be the following:

$$
\begin{aligned}
U(x, t) & =c(\epsilon, \hbar, n) \int_{x_{0}} \int_{p_{0}} \Psi\left(x, t ; x_{0}, p_{0}\right) \delta\left(p_{0}-\tilde{\tau}_{x}\left(x_{0}\right)\right) d p_{0} d x_{0} \\
& =c(\epsilon, \hbar, n) \int_{x_{0}} \tilde{\Psi}\left(x, t ; x_{0}\right) d x_{0}
\end{aligned}
$$

where

$$
\begin{aligned}
\tilde{\Psi}\left(x, t ; x_{0}\right) & =A\left(x, t ; x_{0}\right) \exp \left[i \frac{\tau\left(x, t ; x_{0}\right)}{\hbar}\right] \\
A\left(x, t ; x_{0}\right) & =A\left(t ; x_{0}\right) \\
\tau\left(x, t ; x_{0}\right) & =\tau\left(t ; x_{0}\right)+p\left(t ; x_{0}\right) \cdot\left(x-x\left(t ; x_{0}\right)\right)+\frac{1}{2}\left(x-x\left(t ; x_{0}\right)\right)^{T}\left(B C^{-1}\right)\left(x-x\left(t ; x_{0}\right)\right) .
\end{aligned}
$$

The following lemma proved in [35] holds in terms of recovering the initial data by the initial beam summation:

Lemma 2.3. Let $\phi_{0} \in C^{\infty}\left(\mathbb{R}^{n}\right)$ be a real-valued function and $a_{0} \in C_{0}^{\infty}\left(\mathbb{R}^{n}\right)$. Define

$$
\begin{aligned}
u(x) & =a_{0}(x) \exp \left[\frac{i}{\hbar} \phi_{0}(x)\right] \\
v(x ; y) & =\left(\frac{\epsilon}{2 \pi \hbar}\right)^{\frac{n}{2}} a_{0}(y) \exp \left\{\frac{i}{\hbar}\left[\phi_{0}(y)+\phi_{0}^{\prime}(y)(x-y)\right]+\frac{1}{2 \hbar}\left[i \phi_{0}^{\prime \prime}(y)-\epsilon\right](x-y)^{2}\right\} .
\end{aligned}
$$

Then

$$
\left\|u(x)-\int_{\mathbb{R}^{n}} v(x ; y) d y\right\|_{L^{2}} \leq C\left(\frac{\hbar}{\epsilon}\right)^{1 / 2}
$$

for some constant $C$. 
The parameter $\epsilon$ in the beam decomposition controls the initial beam width since the amplitude of the beam decays away from the center in the order of

$$
O\left(\exp \left[\frac{-\epsilon}{2 \hbar}(x-y)^{2}\right]\right)
$$

Theoretically, this parameter will not affect the asymptotic solution as $\hbar \rightarrow 0$ and therefore we can arbitrarily pick this width. One simple way is to pick unity. In this case, as seen from the estimate, the initial condition of this particular asymptotic decomposition converges to the exact initial profile in the order of $O\left(\hbar^{1 / 2}\right)$.

In numerical implementation, numerical quadrature for the beam integral will introduce errors as well. Suppose that we shoot out rays parameterized by $y$ with a uniform spacing $\Delta x$, and we approximate the integral by a simple midpoint quadrature. Then the overall error in approximating the initial wave function will be $O\left(\left(\frac{\hbar}{\epsilon}\right)^{1 / 2}+\Delta x^{2}\right)$. If $\epsilon=1$, then the error is $O\left(\hbar^{1 / 2}+\Delta x^{2}\right)$; in other words, if $\hbar$ is fixed and we increase the number of beams by letting $\Delta x \rightarrow 0$, then the approximation error in the initial wave function is still in the order of $\hbar^{1 / 2}$, which is undesirable.

Here we propose to balance the two errors by choosing the initial beam width $\epsilon$ according to $\hbar$ and $\Delta x$. Consider one Gaussian centered at zero in the form of $\exp \left(-\epsilon y^{2} / 2 \hbar\right)$, which has the standard deviation of $\sigma=\sqrt{\hbar / \epsilon}$. Since this Gaussian decays to almost zero $3 \sigma$ away from the center, we propose to resolve this Gaussian using a fixed number of grid points. Numerically, we use three grid points to resolve $3 \sigma$, which gives $\epsilon=\hbar / \Delta x^{2}$. Another motivation of this particular choice comes from [12] in which the author makes use of a sum of shifted Gaussians to approximate unity,

$$
1 \simeq \frac{1}{\sqrt{2 \pi}} \frac{\Delta x}{\sigma} \sum_{i} \exp \left(-\frac{\left(x-x_{i}\right)^{2}}{2 \sigma^{2}}\right),
$$

where $x_{i}=i \Delta x, i=0, \pm 1, \pm 2, \cdots$. The conclusion leads to a restriction that $\Delta x<2 \sigma$. Relating this condition to $\epsilon$, we have $\epsilon<4 \hbar / \Delta x^{2}$. Our choice indeed satisfies this extra accuracy requirement according to [12]. Similar considerations can also be found in [10,33].

With this particular choice of the initial beam width, we have the following error estimate in the initial wave decomposition,

$$
\left\|u(x)-\Delta x \sum_{i} v\left(x ; x_{i}\right)\right\|_{L^{2}} \leq C \Delta x,
$$

where $C$ is a constant. Given $\hbar$ and $\epsilon$, this gives a reasonable numerical recipe for decomposing an initial wave function into Gaussians.

We remark that we may set up a mesh which is fine enough so that it will resolve Gaussians for $\hbar$ larger than a certain $\hbar_{\text {min }}$. As a result, we may not recompute necessary beam ingredients; this is essentially done in [17] for the Helmholtz equation in the high frequency regime.

\subsubsection{Initializing by pointwise matching decomposition (PM)}

Because the amplitude function $A_{0}$ has compact support, we can further improve the numerical accuracy in the initial decomposition by requiring that the summation matches with the initial wave 
function pointwise at the discrete level. Mathematically, this means that we determine $A\left(x_{i}, 0\right)$ for all $i$ such that

$$
U\left(x_{j}, 0\right)=\Delta x \sum_{i} A\left(x_{i}, 0\right) \exp \left(\frac{i \tau_{0}\left(x_{j} ; x_{i}\right)}{\hbar}\right)
$$

for all $j$ with

$$
\tau_{0}\left(x ; x_{i}\right)=\tilde{\tau}_{0}\left(x_{i}\right)+\frac{\partial \tilde{\tau}_{0}\left(x_{i}\right)}{\partial x}\left(x-x_{i}\right)+\frac{1}{2}\left(x-x_{i}\right)^{T} \frac{\partial^{2} \tilde{\tau}_{0}\left(x_{i}\right)}{\partial x^{2}}\left(x-x_{i}\right)+i \frac{1}{2} \epsilon\left(x-x_{i}\right)^{2} .
$$

Letting $A_{i}=A\left(x_{i}, 0\right)$ and $f_{i}=U\left(x_{i}, 0\right)$, we obtain $A_{i}$ by solving the following system of linear equations for $\overrightarrow{\mathcal{A}}=\left(A_{1}, \cdots, A_{I}\right)^{T}$

$$
\underline{\mathcal{E}} \overrightarrow{\mathcal{A}}=\overrightarrow{\mathcal{F}}
$$

where $\overrightarrow{\mathcal{F}}=\left(f_{1}, \cdots, f_{I}\right)^{T}, \underline{\mathcal{E}}=\left[\mathcal{E}_{i, j}\right]$ and $\mathcal{E}_{i, j}=\exp \left[i \tau_{0}\left(x_{j} ; x_{i}\right) / \hbar\right] \Delta x$. Following the same argument as in the previous section, we use $\epsilon=O\left(\hbar / \Delta x^{2}\right)$ which resolves the Gaussian using a fixed number of grid points.

A similar decomposition has been widely used in the radial basis function method for multivariate approximation. We refer interested readers to [3] for more analysis on properties of the matrix $\underline{\mathcal{E}}$. In terms of a more general framework, these coefficients $\overrightarrow{\mathcal{A}}$ can be found using the following projection such that for $k=1, \cdots, I$

$$
\int U\left(x_{j}, 0\right) \exp \left(\frac{i \tau_{0}\left(x_{j} ; x_{k}\right)}{\hbar}\right) d x_{j}=\int \Delta x \sum_{i} A\left(x_{i}, 0\right) \exp \left(\frac{i \tau_{0}\left(x_{j} ; x_{i}\right)}{\hbar}\right) \exp \left(\frac{i \tau_{0}\left(x_{j} ; x_{k}\right)}{\hbar}\right) d x_{j} \text {. }
$$

\subsubsection{Initializing by semi-classical Fourier transform}

Applying the semi-classical Fourier transform to the initial wave function $U(x, 0)=U_{0}(x)$, we have

$$
\hat{U}(p, 0 ; \hbar)=\frac{1}{(2 \pi \hbar)^{n / 2}} \int_{-\infty}^{+\infty} U_{0}(x) \exp \left(\frac{-i p \cdot x}{\hbar}\right) d x .
$$

Then the following system will be solved to obtain ingredients for beam propagation:

$$
\begin{aligned}
\dot{x} & =H_{p},\left.\quad x\right|_{t=0}=x_{0}, \\
\dot{p} & =-H_{x},\left.\quad p\right|_{t=0}=p_{0}, \\
\dot{\tau} & =\frac{1}{2} p^{2}-V(x),\left.\quad \tau\right|_{t=0}=x_{0} \cdot p_{0} \\
\dot{B} & =-H_{x p}^{T} B-H_{x x} C,\left.\quad B\right|_{t=0}=i \epsilon I \\
\dot{C} & =H_{p p} B+H_{p x} C,\left.\quad C\right|_{t=0}=I \\
\dot{A} & =-\frac{1}{2} \operatorname{trace}\left(B C^{-1}\right) A,\left.\quad A\right|_{t=0}=\hat{U}\left(p_{0}, 0 ; \hbar\right) .
\end{aligned}
$$

Thus, the asymptotic solution to the Schrödinger equation is obtained by integrating all the beams parametrized by the initial point $\left(x_{0}, p_{0}\right)$,

$$
U(x, t)=\left(\frac{\epsilon^{1 / 2}}{2 \pi \hbar}\right)^{n} \int_{p_{0}} \int_{x_{0}} \Psi\left(x, t ; x_{0}, p_{0}\right) d x_{0} d p_{0}
$$


Eulerian Gaussian Beams for Schrödinger Equations in the Semi-Classical Regime

where $\Psi$ is the contribution from a single Gaussian beam defined as in (19).

It is easy to verify that at $t=0$ we recover the initial data in the case when the initial phase function is linear in $x$. Since

$$
\Psi\left(x, 0 ; x_{0}, p_{0}\right)=\hat{U}\left(p_{0}, 0\right) \exp \left(i \frac{\tau\left(x, 0 ; x_{0}, p_{0}\right)}{\hbar}\right)
$$

where

$$
\tau\left(x, 0 ; x_{0}, p_{0}\right)=x_{0} \cdot p_{0}+p_{0} \cdot\left(x-x_{0}\right)+\frac{i \epsilon}{2}\left(x-x_{0}\right)^{T}\left(x-x_{0}\right),
$$

we have

$$
\begin{aligned}
U(x, 0) & =\left(\frac{\epsilon^{1 / 2}}{2 \pi \hbar}\right)^{n} \int_{p_{0}} \int_{x_{0}} \Psi\left(x, 0 ; x_{0}, p_{0}\right) d x_{0} d p_{0} \\
& =\left(\frac{\epsilon^{1 / 2}}{2 \pi \hbar}\right)^{n} \int_{p_{0}} \int_{x_{0}} \hat{U}\left(p_{0}, 0\right) \exp \left[i\left(\frac{p_{0} \cdot x+\frac{i \epsilon}{2}\left|x-x_{0}\right|^{2}}{\hbar}\right)\right] d x_{0} d p_{0} \\
& =\frac{1}{(2 \pi \hbar)^{n / 2}} \int_{p_{0}} \hat{U}\left(p_{0}, 0\right) \exp \left(i \frac{p_{0} \cdot x}{\hbar}\right) d p_{0} \\
& =U_{0}(x) .
\end{aligned}
$$

A similar initialization has been used in modeling mountain waves in [36].

\subsubsection{Initializing by the Fourier-Bros-Iagolnitzer (FBI) transform}

The Fourier-Bros-Iagolnitzer (FBI) transform [22] provides another approach for decomposing the initial data into Gaussians. The FBI transform is defined by the following formula:

$$
\hat{U}(x, p ; \hbar)=\mathcal{T} U_{0}=\alpha_{n, \hbar} \exp \left(-\frac{p^{2}}{2 \hbar}\right) \int_{y} \exp \left[-\frac{(x-i p-y)^{2}}{2 \hbar}\right] U_{0}(y) d y,
$$

with the normalization constant given by

$$
\alpha_{n, \hbar}=2^{-n / 2}(\pi \hbar)^{-3 n / 4} .
$$

The main advantage of the FBI transform over the Fourier transform is that this transform can provide information simultaneously about the local behavior of $U_{0}$ and that of its semi-classical Fourier transform, namely, the microlocal behavior of the function $U_{0}$ [22]. Therefore, the FBI transform provides a micro-localized representation for $U_{0}$ in phase space so that the total number of beams in phase space can be reduced as we will see in numerical examples.

To construct beams, we solve the following system to obtain ingredients for beam propagation:

$$
\begin{aligned}
\dot{x} & =H_{p},\left.\quad x\right|_{t=0}=x_{0}, \\
\dot{p} & =-H_{x},\left.\quad p\right|_{t=0}=p_{0}, \\
\dot{\tau} & =\frac{1}{2} p^{2}-V(x),\left.\quad \tau\right|_{t=0}=0, \\
\dot{B} & =-H_{x p}^{T} B-H_{x x} C,\left.\quad B\right|_{t=0}=i I, \\
\dot{C} & =H_{p p} B+H_{p x} C,\left.\quad C\right|_{t=0}=I, \\
\dot{A} & =-\frac{1}{2} \operatorname{trace}\left(B C^{-1}\right) A,\left.\quad A\right|_{t=0}=\hat{U}\left(x_{0}, p_{0} ; \hbar\right) .
\end{aligned}
$$


Eulerian Gaussian Beams for Schrödinger Equations in the Semi-Classical Regime

The asymptotic solution to the Schrödinger equation is obtained by integrating all the beams parametrized by the initial point $\left(x_{0}, p_{0}\right)$,

$$
U(x, t)=\alpha_{n, \hbar} \int_{x_{0}} \int_{p_{0}} \Psi\left(x, t ; x_{0}, p_{0}\right) d x_{0} d p_{0} .
$$

It is easy to show that at $t=0$ we recover the initial data. Since

$$
\Psi\left(x, 0 ; x_{0}, p_{0}\right)=\hat{U}\left(x_{0}, p_{0} ; \hbar\right) \exp \left(i \frac{\tau\left(x, 0 ; x_{0}, p_{0}\right)}{\hbar}\right)
$$

where

$$
\tau\left(x, 0 ; x_{0}, p_{0}\right)=p_{0} \cdot\left(x-x_{0}\right)+\frac{i}{2}\left(x-x_{0}\right)^{T}\left(x-x_{0}\right),
$$

we have

$$
\begin{aligned}
U(x, 0) & =\alpha_{n, \hbar} \int_{x_{0}} \int_{p_{0}} \Psi\left(x, 0 ; x_{0}, p_{0}\right) d x_{0} d p_{0} \\
& =\alpha_{n, \hbar} \int_{x_{0}} \int_{p_{0}} \hat{U}\left(x_{0}, p_{0} ; \hbar\right) \exp \left[-\frac{i}{\hbar}\left(x_{0}-x\right) \cdot p_{0}-\frac{\left(x_{0}-x\right)^{2}}{2 \hbar}\right] d x_{0} d p_{0} \\
& =\mathcal{T}^{*} \mathcal{T} U_{0} \\
& =U_{0} .
\end{aligned}
$$

Here we have used a property of FBI transform [22]: $T^{*} T=I$ under some appropriate conditions.

To the best of our knowledge, initializing beam propagation by the FBI transform is new.

\subsection{Numerical Methods}

\subsubsection{A Numerical FBI Transform}

For some simple cases, we can analytically compute the integral (39). However, it is generally difficult to find the closed form of $\hat{U}$ for an arbitrary $U_{0}$. In this section, we will discuss how to numerically evaluate the integral. In fact, the FBI transform is related closely to the Gabor transform

$$
G_{U_{0}(x)}(x, p)=\int_{-\infty}^{\infty} \exp \left(-\pi(y-x)^{2}\right) \exp (-2 \pi i p \cdot y) U_{0}(y) d y
$$

by

$$
\hat{U}(x, p, \hbar)=\alpha_{n, \hbar} \sqrt{2 \pi \hbar} \exp \left(\frac{i x \cdot p}{\hbar}\right) G_{U_{0}(x / \sqrt{2 \pi \hbar})}(\sqrt{2 \pi \hbar} x, \sqrt{2 \pi \hbar} p) .
$$

It is still an active research area to develop a fast discrete Gabor transform [31, 37]. Therefore in this paper, instead of applying these newly developed methods, we simply implement the following $O\left(n^{3}\right)$ algorithm to embed the one-dimensional initial wave function in the phase space using the FBI transform.

We assume that $U_{0}$ has compact support and we denote $U_{i}=U_{0}\left(x_{i}\right)$ at the grid points $x_{i}$ for $i=1, \cdots, I$. Consider an equivalent form of the FBI transform,

$$
\hat{U}=\mathcal{T} U_{0}=\alpha_{n, \hbar} \int_{y} \exp \left[-\frac{(x-y)^{2}}{2 \hbar}+\frac{p \cdot(x-y) i}{\hbar}\right] U_{0}(y) d y,
$$


One way to determine $\hat{U}\left(x_{i^{\prime}}, p_{j^{\prime}} ; \hbar\right)$ is to approximate the above integral using the midpoint quadrature,

$$
\hat{U}\left(x_{i^{\prime}}, p_{j^{\prime}} ; \hbar\right)=\alpha_{n, \hbar} \Delta x \sum_{j=1}^{I} \exp \left[-\frac{\left(x_{i^{\prime}}-x_{j}\right)^{2}}{2 \hbar}+\frac{p_{j^{\prime}} \cdot\left(x_{i^{\prime}}-x_{j}\right) i}{\hbar}\right] U_{j}
$$

for each individual $\left(x_{i^{\prime}}, p_{j^{\prime}}\right)$. In MATLAB, we do not implement this in a point-by-point fashion. For a one dimensional numerical FBI transform, we first construct two matrices $A$ and $B$ with each entry given by $-\left(x_{i^{\prime}}-x_{j}\right)^{2} / 2 \hbar$ and $i\left(x_{i^{\prime}}-x_{j}\right) / \hbar$, respectively. These two matrices are independent of $p_{j^{\prime}}$ and are stored separately from the integration routine. Then, for each $p_{j^{\prime}}$ we construct the matrix $\exp \left(\mathrm{A}+p_{j^{\prime}} \mathrm{B}\right)$ and multiply it by the vector u containing $U_{0}\left(x_{i^{\prime}}\right)$. This gives $\hat{U}\left(x_{i^{\prime}}, p_{j^{\prime}} ; \hbar\right)$ for all $i^{\prime}=1, \cdots, I$ for a fixed $p_{j^{\prime}}$.

The above approximation converges to the exact solution as $\Delta x \rightarrow 0$. However, in practice, we have to avoid the aliasing error as in the numerical Fourier transform. For simplicity, we consider the one-dimensional case where $U_{0}(y)$ is real. We first rewrite (39) into

$$
\mathcal{T} U_{0}=\alpha_{n, \hbar} \exp \left(\frac{i x p}{\hbar}\right) \int_{y} \exp \left[-\frac{(x-y)^{2}}{2 \hbar}\right] \exp \left[-\frac{i y p}{\hbar}\right] U_{0}(y) d y
$$

To well-sample the oscillations from $\exp (-i y p / \hbar)$, we require

$$
\frac{\max \left(-p_{\min }, p_{\max }\right) \Delta x}{\hbar}<\frac{\pi}{2}
$$

which implies $p \in(-\hbar \pi / 2 \Delta x, \hbar \pi / 2 \Delta x)$.

One possible improvement to the current approach is to truncate the Gaussians in the kernel and limit the evaluation of the integral in a small neighborhood of each $x$. For instance, the matrix $\exp (\mathrm{A})$ can be approximated by a sparse matrix by ignoring those off-diagonal entries when $\left(x_{i^{\prime}}-x_{j}\right)$ is significantly large. Presumably, this will reduce the computational complexity from $O\left(n^{3}\right)$ to $O\left(n^{2} \log n\right)$; however, this is left for future work.

\subsubsection{Algorithm: LGB-AD}

Here we summarize the Lagrangian Gaussian Beam summation algorithm for solving the onedimensional Schrodinger equation by initializing beams based on the asymptotic decomposition (AD). It is relatively straight-forward to generalize the algorithm to higher dimensions or use different initialization for beam propagation.

Algorithm 1: LGB-AD

1. Discretize the computational domain

$$
\begin{aligned}
x_{i}=x_{\min }+(i-1) \Delta x & , \quad i=1,2, \cdots, I \\
p_{i}=p\left(x_{i}\right)=\tau_{0}^{\prime}\left(x_{i}\right) & , \quad i=1,2, \cdots, I \\
t_{k}=t_{0}+k \Delta t & , \quad k=1,2, \cdots, K,
\end{aligned}
$$

where $\Delta x=\left(x_{\max }-x_{\min }\right) /(I-1)$ and $\Delta t=\left(t_{f}-t_{0}\right) / K$. Initialize $\epsilon$. 
2. At a fixed $t=t_{k}$, for each $i^{\prime}=1, \cdots, I$,

(a) solve the ray tracing system (11-16) if $A_{0}\left(x_{i^{\prime}}\right) \neq 0$ using the initial conditions

$$
\begin{aligned}
x\left(t=t_{0}\right) & =x_{i^{\prime}} \\
p\left(t=t_{0}\right) & =p_{i^{\prime}} \\
\tau\left(t=t_{0}\right) & =\tilde{\tau}_{0}^{\prime}\left(x_{i^{\prime}}\right) \\
A\left(t=t_{0}\right) & =A_{0}\left(x_{i^{\prime}}\right) \\
B\left(t=t_{0}\right) & =\frac{\partial^{2} \tau_{0}\left(x_{i^{\prime}}\right)}{\partial x^{2}}+i \epsilon I \\
C\left(t=t_{0}\right) & =I .
\end{aligned}
$$

(b) for each $\tilde{i}=1, \cdots, \tilde{I}$, compute

$$
\Psi\left(x_{\tilde{i}}, t_{k} ; x_{i^{\prime}}, p_{i^{\prime}}\right)=A\left(t_{k}\right) \exp \left(\frac{i \tau\left(x_{\tilde{i}}, t_{k}\right)}{\hbar}\right)
$$

where

$$
\tau\left(x_{\tilde{i}}, t_{k}\right)=\tau\left(t_{k}\right)+p\left(t_{k}\right)\left(x_{\tilde{i}}-x\left(t_{k}\right)\right)+\frac{1}{2}\left(x_{\tilde{i}}-x\left(t_{k}\right)\right)^{T}\left(B\left(t_{k}\right) C\left(t_{k}\right)^{-1}\right)\left(x_{\tilde{i}}-x\left(t_{k}\right)\right) .
$$

3. Sum up the individual wave function. For each $\tilde{i}=1, \cdots, \tilde{I}$,

$$
U\left(x_{\tilde{i}}, t_{k}\right)=c(\epsilon, \hbar, n) \Delta x \sum_{i^{\prime}=1}^{I} \Psi\left(x_{\tilde{i}}, t_{k} ; x_{i^{\prime}}, p_{i^{\prime}}\right) .
$$

Theoretically, (17) is the exact solution to the transport equation for the amplitude. However, since $C\left(t ; x_{0}, p_{0}\right)$ is complex, we have to choose the correct branch for the square root in (17) so that $A\left(t ; x_{0}, p_{0}\right)$ is continuous along the characteristics. Therefore, it is easier to directly solve (16) which will automatically determine the continuous solution in time.

As described above, we approximate the integral in the Gaussian beam summation using the midpoint quadrature. We discretize the $x$-direction of the phase space using a mesh with grid size $\Delta x$. This resolution is chosen solely according to the accuracy in the solution. The finer the discretization, the better the approximation will be in calculating the Gaussian summation integral using the midpoint rule. Further, in the cases when we pick $\epsilon=\hbar / \Delta x^{2}$, this mesh size also contributes an error of $O(\Delta x)$ to the asymptotic decomposition of the initial wave function. This accuracy requirement is different from the resolution requirement. To visualize the solution, we have to sample the continuous solution $U(x, t),(20)$, defined for all $x \in \mathbb{R}^{n}$. These sampling locations might be different from those grid locations we used to discretize the $x$-direction in the phase space. In general, both the number and the locations of these sampling points can be arbitrary. However, to resolve fine oscillations in the solution, we have to sample the solution on a mesh which is finer than the scale of those oscillations. This is a requirement imposed only on the visualizing step. To distinguish these sampling points from the discretization points $x_{i}$, we have denoted them by $x_{\tilde{i}}$ in the above algorithm. 
When we initialize beam propagation in phase space using one of the transforms, we only modify the above algorithm by discretizing the $p$-direction using $p_{j}=p_{\min }+(j-1) \Delta p$, then shoot more rays, and sum more beams with a summation of $j^{\prime}$ from 1 to $J$ in the last step.

In terms of numerical implementation, the initial beams in the Lagrangian formulation are uniformly distributed over phase space, while the beam locations at the final time are not uniformly distributed. Since we have no control of these beam locations, it is generally difficult to determine the size of the computational domain and the total number of beams used.

\section{Eulerian Gaussian Beams (EGB)}

\subsection{Formulations}

By the level set methodology we embed the ray tracing system into the Liouville equation in phase space. Let $\phi(x, p, t) \in \mathbb{R}^{n}, \psi(x, p, t) \in \mathbb{R}^{n}$, and $T(x, p, t) \in \mathbb{R}^{1}$. We have the following level set equations and the phase equation,

$$
\begin{aligned}
\phi_{t}+H_{p} \cdot \phi_{x}-H_{x} \cdot \phi_{p} & =0, \quad \phi(x, p, 0)=x \\
\psi_{t}+H_{p} \cdot \psi_{x}-H_{x} \cdot \psi_{p} & =0, \quad \psi(x, p, 0)=p \\
T_{t}+H_{p} \cdot T_{x}-H_{x} \cdot T_{p} & =\frac{1}{2} p^{2}-V(x), \quad T(x, p, 0)=\tau_{0}(x) .
\end{aligned}
$$

Similarly we have the equations for $A, B$ and $C$.

$$
\begin{aligned}
A_{t}+H_{p} \cdot A_{x}-H_{x} \cdot A_{p} & =-\frac{1}{2} \operatorname{trace}\left(B C^{-1}\right) A,\left.\quad A\right|_{t=0}=A_{0}(x, p), \\
B_{t}+H_{p} \cdot B_{x}-H_{x} \cdot B_{p} & =-H_{x p}^{T} B-H_{x x} C,\left.\quad B\right|_{t=0}=B_{0}(x, p), \\
C_{t}+H_{p} \cdot C_{x}-H_{x} \cdot C_{p} & =H_{p p} B+H_{p x} C,\left.\quad C\right|_{t=0}=I .
\end{aligned}
$$

The initial conditions for functions $A$ and $B$ are specified according to how the initial wave function is decomposed into a summation of Gaussians. If we initialize beam propagation by the FBI transform, then we set $B_{0}(x, p)=i I$ and $A_{0}(x, p)=\hat{U}(x, p ; \hbar)$. If we initialize beam propagation by the asymptotic decomposition or the pointwise matching decomposition, then we set $B_{0}(x, p)=\frac{\partial^{2} \tilde{\tau}_{0}}{\partial x^{2}}(x)+i \epsilon I$ and $A_{0}(x, p)=A_{0}(x)$.

Now we have all the ingredients for constructing Eulerian Gaussian beams. If the beam propagation is initialized by the Fourier transform or the FBI transform, the asymptotic solution is given by

$$
U(x, t)=c(\epsilon, \hbar, n) \int_{p^{\prime}} \int_{x^{\prime}} \Psi\left(x, t ; x^{\prime}, p^{\prime}\right) \Delta\left(x_{0}, p_{0} ; x^{\prime}, p^{\prime}, t\right) d x^{\prime} d p^{\prime}
$$

where

$$
\begin{aligned}
\Psi\left(x, t ; x^{\prime}, p^{\prime}\right) & =A\left(x^{\prime}, p^{\prime}, t\right) \exp \left[i \frac{1}{\hbar} \tau\left(x, t ; x^{\prime}, p^{\prime}\right)\right] \\
\tau\left(x, t ; x^{\prime}, p^{\prime}\right) & =T\left(x^{\prime}, p^{\prime}, t\right)+p^{\prime} \cdot\left(x-x^{\prime}\right)+\frac{1}{2}\left(x-x^{\prime}\right)^{T}\left(B C^{-1}\right)\left(x-x^{\prime}\right),
\end{aligned}
$$


and $\Delta\left(x_{0}, p_{0} ; x^{\prime}, p^{\prime}, t\right)$ is the Jacobian of the map $\left(x^{\prime}, p^{\prime}\right) \rightarrow\left(x_{0}, p_{0}\right)$, where $\left(x_{0}, p_{0}\right)$ refers to the initial values of the level sets $\phi$ and $\psi$ arriving at $\left(x^{\prime}, p^{\prime}\right)$ at time $t$. Moreover, the Jacobian of the map $\left(x_{0}, p_{0}\right) \rightarrow\left(x^{\prime}, p^{\prime}\right)$ satisfies the conservation law

$$
\Delta_{t}+\left(H_{p} \Delta\right)_{x}-\left(H_{x} \Delta\right)_{p}=0, \Delta_{t=0}=1,
$$

and it follows that $\Delta\left(x_{0}, p_{0} ; x^{\prime}, p^{\prime}, t\right) \equiv 1$. This implies that the asymptotic solution is simply given by

$$
U(x, t)=c(\epsilon, \hbar, n) \int_{p^{\prime}} \int_{x^{\prime}} \Psi\left(x, t ; x^{\prime}, p^{\prime}\right) d x^{\prime} d p^{\prime} .
$$

On the other hand, if the beam propagation is initialized by the asymptotic decomposition or the pointwise matching decomposition, we extract the necessary information by looking into the zero level set defined by

$$
\left\{(x, p): \psi(x, p, t)-\partial \tilde{\tau}_{0}[\phi(x, p, t)] / \partial x=0\right\} .
$$

For simplicity of notations, we will below use $\tilde{\tau}_{0}^{\prime}$ to denote $\frac{\partial \tilde{\tau}_{0}}{\partial x}$. Then the wave function can be computed by

$$
U(x, t)=c(\epsilon, \hbar, n) \int_{p^{\prime}} \int_{x^{\prime}} \Psi\left(x, t ; x^{\prime}, p^{\prime}\right) \delta\left[\psi\left(x^{\prime}, p^{\prime}, t\right)-\tilde{\tau}_{0}^{\prime}\left(\phi\left(x^{\prime}, p^{\prime}, t\right)\right)\right] d x^{\prime} d p^{\prime},
$$

To avoid discretizing the $\delta$-function, we will use the properties of the $\delta$-function to simplify the above integral; such a technique has been used in [4]. We will first consider the case of $n=1$, and we will comment on the case of $n \geq 2$. If $n=1$, we first explicitly determine the set of points where the zero level set intersects with the grid lines, i.e.

$$
\Gamma=\left\{(x, p): \psi\left(x_{i}, p\right)=\tilde{\tau}_{0}^{\prime}\left[\phi\left(x_{i}, p\right)\right] \text { or } \psi\left(x, p_{j}\right)=\tilde{\tau}_{0}^{\prime}\left[\phi\left(x, p_{j}\right)\right] \text { for } i=1, \cdots, I, j=1, \cdots, J .\right\} ;
$$

then we interpolate all necessary ingredients at these locations and the wave function can be integrated by

$$
U(x, t)=c(\epsilon, \hbar, n) \sum_{\Gamma} \Delta \phi(\Gamma) \Psi(x, t ; \Gamma),
$$

where the weight $\Delta \phi(\Gamma)$ (the Jacobian) is computed by taking the difference in the take-off value of $x$ between two adjacent beams, as shown in Figure 1. A similar approach has been developed in [17] in the context of solving the Helmholtz equation in the high frequency regime. Such an approach can be generalized to $n \geq 2$ in the following way. Assuming that $x \in \mathbb{R}^{n}$, we first determine a set of points $\Gamma$ in phase space $\mathbb{R}^{2 n}$ which samples the zero level set. For example, this can be done by the isosurfacing algorithm presented in [26]. In higher dimensions, since the initial implicit surface $\left\{(x, p): p=\nabla \tilde{\tau_{0}}(x)\right\}$ can be parameterized by $x \in \mathbb{R}^{n}$, we can apply the summation formula (66) accordingly.

We remark that even though the Lagrangian formulation (20) and the Eulerian formulation (62) are theoretically equivalent to each other, there are multiple advantages of the above Eulerian formulation. The first advantage is that we have uniform resolution of beam distribution, so that the Gaussian beam summation will have uniform resolution as well. The second advantage is that 


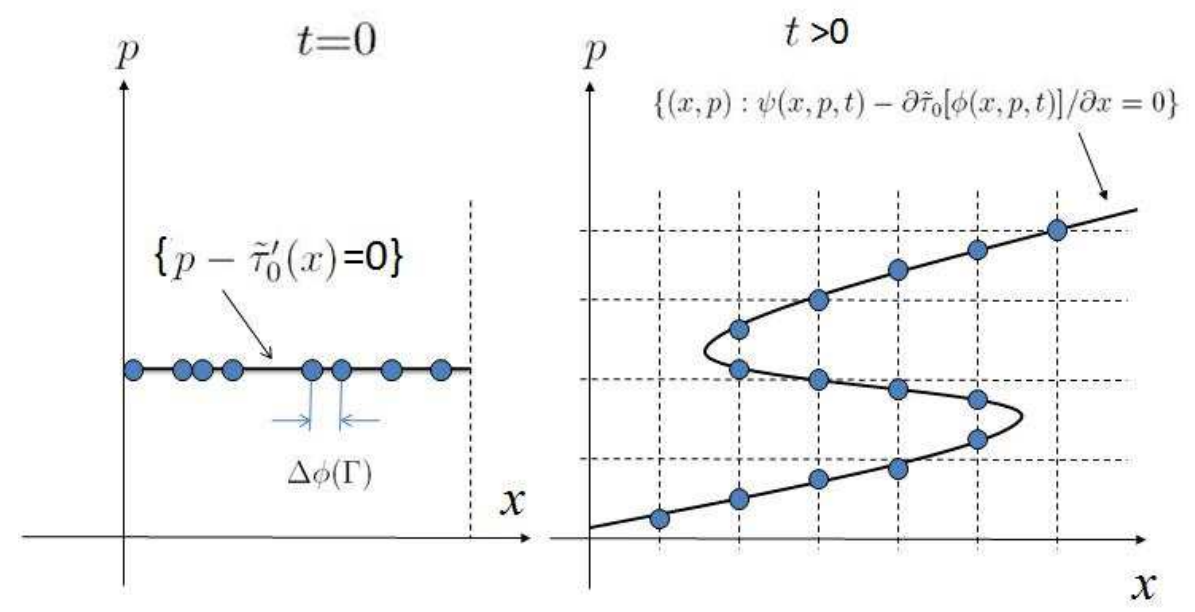

Figure 1: Eulerian Gaussian Beam with the initial wave function decomposed using the asymptotic decomposition or the pointwise matching decomposition. The set of sampling points $\Gamma$ are shown in blue dots. The useful level set is plotted using a black solid line.

we can generate wave functions under the same external potential with different initial profiles by slightly modifying the solution from the amplitude equation in (57). The idea is to use Lemma 2.2 which essentially means that the amplitude at $t>0$ is proportional to the initial condition $A_{0}$. Using this relation, we have

$$
A^{\text {new }}\left(x_{i}, p_{j}, t_{k}\right)=A^{\text {old }}\left(x_{i}, p_{j}, t_{k}\right) \frac{A_{0}^{\text {new }}\left(\phi\left(x_{i}, p_{j}, t_{k}\right), \psi\left(x_{i}, p_{j}, t_{k}\right)\right)}{A_{0}^{\text {old }}\left(\phi\left(x_{i}, p_{j}, t_{k}\right), \psi\left(x_{i}, p_{j}, t_{k}\right)\right)},
$$

where $A^{\text {old }}$ is the solution obtained by using the initial condition $A_{0}^{\text {old }}$ from the previous initial wave function, and $A^{\text {new }}$ is the new amplitude solution with a different initial wave function.

\subsection{A Preliminary Numerical Method}

We give the Eulerian Gaussian Beam summation algorithm for constructing wave functions when the beam propagation is initialized by either the Fourier transform or the FBI transform. It is straight-forward to generalize this algorithm to other initializations or problems in higher dimensions.

Algorithm 2: EGB-Tran-PDE:

1. Discretize the computational domain

$$
\begin{aligned}
& x_{i}=x_{\min }+(i-1) \Delta x \quad, \quad \Delta x=\frac{x_{\max }-x_{\min }}{I-1}, i=1,2, \cdots, I \\
& p_{j}=p_{\min }+(j-1) \Delta p \quad, \quad \Delta p=\frac{p_{\max }-p_{\min }}{J-1}, j=1,2, \cdots, J
\end{aligned}
$$




$$
t_{k}=t_{0}+k \Delta t \quad, \quad \Delta t=\frac{t_{f}-t_{0}}{K}, k=1,2, \cdots, K,
$$

and initialize $\epsilon$ and all functions $\phi_{i, j, 0}, \psi_{i, j, 0}, T_{i, j, 0}, A_{i, j, 0}, B_{i, j, 0}$ and $C_{i, j, 0}$ according to (56-57).

2. Solve the Liouville equations according to (56-57). For each $i=1, \cdots, I, j=1, \cdots, J$ and $k=2, \cdots, K$, determine

$$
\phi\left(x_{i}, p_{j}, t_{k}\right), \psi\left(x_{i}, p_{j}, t_{k}\right), T\left(x_{i}, p_{j}, t_{k}\right), A\left(x_{i}, p_{j}, t_{k}\right), B\left(x_{i}, p_{j}, t_{k}\right), C\left(x_{i}, p_{j}, t_{k}\right) .
$$

3. Construct the individual wave function. At a fixed $t=t_{k}$, for each $i^{\prime}=1, \cdots, I$ and $j^{\prime}=1, \cdots, J$, compute

$$
\Psi\left(x_{\tilde{i}}, t_{k} ; x_{i^{\prime}}, p_{j^{\prime}}\right)=A\left(x_{i^{\prime}}, p_{j^{\prime}}, t_{k}\right) \exp \left[\frac{i \tau\left(x_{\tilde{i}}, t_{k} ; x_{i^{\prime}}, p_{j^{\prime}}\right)}{\hbar}\right]
$$

where

$$
\begin{aligned}
\tau\left(x_{\tilde{i}}, t_{k} ; x_{i^{\prime}}, p_{j^{\prime}}\right)= & T\left(x_{i^{\prime}}, p_{j^{\prime}}, t_{k}\right)+p_{j^{\prime}} \cdot\left(x_{\tilde{i}}-x_{i^{\prime}}\right) \\
& +\frac{1}{2}\left(x_{\tilde{i}}-x_{i^{\prime}}\right)^{T}\left(B\left(x_{i^{\prime}}, p_{j^{\prime}}, t_{k}\right) C\left(x_{i^{\prime}}, p_{j^{\prime}}, t_{k}\right)^{-1}\right)\left(x_{\tilde{i}}-x_{i^{\prime}}\right) .
\end{aligned}
$$

4. Sum up the individual wave function. For each $\tilde{i}=1, \cdots, \tilde{I}$,

$$
U\left(x_{\tilde{i}}, t_{k}\right)=c(\epsilon, \hbar, n) \Delta x \Delta p \sum_{i^{\prime}=1}^{I} \sum_{j^{\prime}=1}^{J} \Psi\left(x_{\tilde{i}}, t_{k} ; x_{i^{\prime}}, p_{j^{\prime}}\right) .
$$

Unfortunately, this algorithm has two main drawbacks. One is related to the accuracy. To obtain accurate solution of the Liouville equations (56-57), we have to use a very fine computational grid even though those PDEs are solved using a high-order numerical scheme such as WENO5-TVDRK3 [34]. Since the number of computational grids is essentially the same as the number of beams used in sampling the integral, this underlying grid refinement might introduce unnecessary beams to over-sample the solution.

Another drawback is related to the computational efficiency. Since the Liouville equations are hyperbolic type, the marching step size is restricted by the CFL condition. The complexity of the whole algorithm is $O\left(n^{2 d+1}\right)$, where $n$ is the number of grid points in each direction in phase space and $d$ is the dimension of physical space.

\subsection{Semi-Lagrangian Methods (SL)}

To improve accuracy and efficiency of the above algorithm, we follow $[18,17]$ which solve Liouville equations (56-57) using a semi-Lagrangian method. This approach can be easily generalized to higher dimensions. We apply the method of characteristics to the level set equations and the phase equation, giving

$$
\begin{aligned}
\frac{D \phi}{D t} & =0 \\
\frac{D \psi}{D t} & =0 \\
\frac{D T}{D t} & =\frac{1}{2} p^{2}-V(x),
\end{aligned}
$$




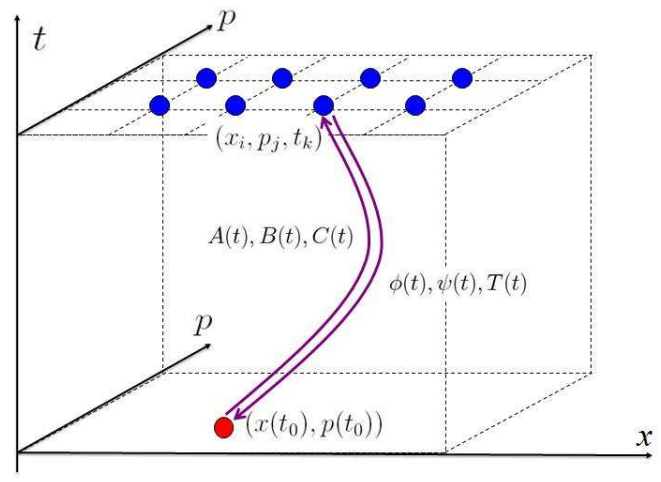

Figure 2: Eulerian Gaussian Beam computed using the semi-Lagrangian method.

where $D / D t$ is the material derivative defined by

$$
\frac{D}{D t}=\frac{\partial}{\partial t}+H_{p} \frac{\partial}{\partial x}-H_{x} \frac{\partial}{\partial p}
$$

At each grid point $\left(x_{i}, p_{j}, t_{k}\right)$ for $i=1, \cdots, I, j=1, \cdots, J$ and $k=2, \cdots, K$ in the phase space, one traces backward from $t=t_{k}$ to $t=t_{0}$ along the characteristic by integrating $\frac{d x}{d t}=H_{p}$ and $\frac{d p}{d t}=-H_{x}$ to obtain $\left(x\left(t_{0}\right), p\left(t_{0}\right)\right)$. For the level set equations, we assign $\phi\left(x_{i}, p_{j}, t_{k}\right)=x\left(t_{0}\right)$ and $\psi\left(x_{i}, p_{j}, t_{k}\right)=p\left(t_{0}\right)$. For the phase equation, we use the reciprocal principle and integrate the source term $\left[p(t)^{2} / 2-V(x(t))\right]$ along the characteristic to obtain $T\left(x_{i}, p_{j}, t_{k}\right)$.

As for $A, B$ and $C$, we apply the method of characteristics and obtain

$$
\begin{aligned}
& \frac{D A}{D t}=-\frac{1}{2} \operatorname{trace}\left(B C^{-1}\right) A,\left.\quad A\right|_{t=0}=A_{0}\left(\phi\left(x_{i}, p_{j}, t_{k}\right), \psi\left(x_{i}, p_{j}, t_{k}\right)\right), \\
& \frac{D B}{D t}=-H_{x p} B-H_{x x} C,\left.\quad B\right|_{t=0}=B_{0}\left(\phi\left(x_{i}, p_{j}, t_{k}\right), \psi\left(x_{i}, p_{j}, t_{k}\right)\right), \\
& \frac{D C}{D t}=H_{p p} B+H_{x p} C,\left.\quad C\right|_{t=0}=C_{0}\left(\phi\left(x_{i}, p_{j}, t_{k}\right), \psi\left(x_{i}, p_{j}, t_{k}\right)\right),
\end{aligned}
$$

with the initial conditions imposed on the level $t=t_{0}$. In this case, we do not have the reciprocal principle as for the phase equation anymore; we need to use the forward ray tracing to solve these quantities along the same characteristic provided by the backward ray tracing. This means that we first compute the ray trajectory by integrating $\frac{d x}{d t}=H_{p}$ and $\frac{d p}{d t}=-H_{x}$ backward in $t$, and then we integrate (75) forward in $t$ along the same characteristic, as shown in Figure 2.

In terms of numerical integration of the Hamiltonian system, we adopt a symplectic scheme. Specifically, to preserves the symplectic structure of the Hamiltonian system,

$$
\begin{aligned}
& \dot{q}=f(q, v) \\
& \dot{v}=g(q, v) .
\end{aligned}
$$


Eulerian Gaussian Beams for Schrödinger Equations in the Semi-Classical Regime

we adopt the following fourth order implicit Runge-Kutta method [5, 9]:

$$
\begin{aligned}
k_{i} & =f\left(q_{n}+h \sum_{j=1}^{s} a_{i j} k_{j}, v_{n}+h \sum_{j=1}^{s} \hat{a}_{i j} l_{j}\right) \\
l_{i} & =g\left(q_{n}+h \sum_{j=1}^{s} a_{i j} k_{j}, v_{n}+h \sum_{j=1}^{s} \hat{a}_{i j} l_{j}\right) \\
q_{n+1} & =q_{n}+h \sum_{i-1}^{s} b_{i} k_{i} \\
v_{n+1} & =v_{n}+h \sum_{i-1}^{s} b_{i} l_{i},
\end{aligned}
$$

where $s=2$ and

$$
\left[a_{i j}\right]=\left[\hat{a}_{i j}\right]=\left(\begin{array}{cc}
1 / 4 & 1 / 4-\sqrt{3} / 6 \\
1 / 4+\sqrt{3} / 6 & 1 / 4
\end{array}\right)
$$

and $b_{i}=1 / 2$.

Therefore, the accuracy in the solution now depends solely on the size of the time step $\Delta z$ but independent of the number of grid points in discretizing the phase space, since we treat each of these grid points independently. This allows us to use relatively small number of beams to sample the solution, which in most cases are sufficient to give reasonable qualitative solution.

Algorithm 3: EGB-Tran-SL:

1. Discretize the computational domain

$$
\begin{aligned}
x_{i}=x_{\min }+(i-1) \Delta x & , \Delta x=\frac{x_{\max }-x_{\min }}{I-1}, i=1,2, \cdots, I \\
p_{j}=p_{\min }+(j-1) \Delta p & , \quad \Delta p=\frac{p_{\max }-p_{\min }}{J-1}, j=1,2, \cdots, J \\
t_{k}=t_{0}+k \Delta t & , \quad \Delta t=\frac{t_{f}-t_{0}}{K}, k=1,2, \cdots, K
\end{aligned}
$$

and initialize $\epsilon$.

2. Solve the Liouville equations backward in time according to (73). For each $i=1, \cdots, I, j=1, \cdots, J$ and $k=2, \cdots, K$, determine

$$
\phi\left(x_{i}, p_{j}, t_{k}\right), \psi\left(x_{i}, p_{j}, t_{k}\right), T\left(x_{i}, p_{j}, t_{k}\right) .
$$

3. Solve the Liouville equations forward in time along the same trajectory $(x(t), p(t), t)$ according to (75). For each $i=1, \cdots, I, j=1, \cdots, J$ and $k=2, \cdots, K$, if $\left|A_{0}\left(x\left(t_{0}\right), p\left(t_{0}\right)\right)\right| \neq 0$, determine

$$
A\left(x_{i}, p_{j}, t_{k}\right), B\left(x_{i}, p_{j}, t_{k}\right), C\left(x_{i}, p_{j}, t_{k}\right) .
$$


4. Construct the individual wave function. At a fixed $t=t_{k}$, for each $i^{\prime}=1, \cdots, I$ and $j^{\prime}=1, \cdots, J$, compute

$$
\Psi\left(x_{\tilde{i}}, t_{k} ; x_{i^{\prime}}, p_{j^{\prime}}\right)=A\left(x_{i^{\prime}}, p_{j^{\prime}}, t_{k}\right) \exp \left(\frac{i \tau\left(x_{\tilde{i}}, t_{k} ; x_{i^{\prime}}, p_{j^{\prime}}\right)}{\hbar}\right)
$$

where

$$
\begin{aligned}
\tau\left(x_{\tilde{i}}, t_{k} ; x_{i^{\prime}}, p_{j^{\prime}}\right)= & T\left(x_{i^{\prime}}, p_{j^{\prime}}, t_{k}\right)+p_{j^{\prime}} \cdot\left(x_{\tilde{i}}-x_{i^{\prime}}\right) \\
& +\frac{1}{2}\left(x_{\tilde{i}}-x_{i^{\prime}}\right)^{T}\left(B\left(x_{i^{\prime}}, p_{j^{\prime}}, t_{k}\right) C\left(x_{i^{\prime}}, p_{j^{\prime}}, t_{k}\right)^{-1}\right)\left(x_{\tilde{i}}-x_{i^{\prime}}\right) .
\end{aligned}
$$

5. Sum up the individual wave function. For each $\tilde{i}=1, \cdots, \tilde{I}$,

$$
U\left(x_{\tilde{i}}, t_{k}\right)=c(\epsilon, \hbar, n) \Delta x \Delta p \sum_{i^{\prime}=1}^{I} \sum_{j^{\prime}=1}^{J} \Psi\left(x_{\tilde{i}}, t_{k} ; x_{i^{\prime}}, p_{j^{\prime}}\right) .
$$

\section{Complexity}

In this section, we will compare the complexities of various methods. In particular, we will concentrate on solving the Schrödinger equation for $s$ different initial wave functions or different $\hbar$ under the same potential. For simplicity, we look at the one-dimensional case, i.e. $d=1$. Let $n$ be the number of beams we used in each $x$ - or $p$-direction. Therefore, the total number of beams is $n$ if we initialize beam propagation by the asymptotic decomposition or the pointwise matching decomposition. The total number of beams is $n^{2}$ if we initialize beam propagation by the FBI transform. Let $N$ be the number of points we used to visualize the solution. In general, we need at least five grid points to visualize one wavelength and this implies $N=O(1 / \hbar)$. Using the asymptotic method, we would like to resolve the solution in much less computational cost so that $n \ll N$.

Since a fast discrete Gabor transform is not yet well-developed, in Tables 1 and 2, we estimate the complexity for the FBI transform as $\beta$ which should be somewhere in between $O\left(n^{2}\right)$ (total number of coefficients) and $O\left(n^{3}\right)$ (our implementation).

When initializing beam propagation by the pointwise matching decomposition, we need to invert a matrix of size $n$-by- $n$. The complexity of an exact direct solver is at most $O\left(n^{3}\right)$. However, since $n \ll N$, the computational cost for this part is not significant in comparison to the cost of the whole algorithm.

Table 1 shows the complexity of the Eulerian Gaussian beam approach. The evolution step involves computing the characteristics (73) and (75). Since we have totally $n^{2}$ grid points and the stability condition requires $\Delta t=O(\Delta x)$, we have $O\left(n^{3}\right)$ operations. When the initial condition is embedded as the zero level set of a level set function, we are required to extract the necessary information. This requires interpolation and root-finding processes which has a complexity of $O(n \log n)$. Since the FBI transform embeds the useful information everywhere, we do not need to extract anything from the function $\phi$ and $\psi$. In the summation process, we compute the contributions to $N$ points from each of these $O(n \log n)$ or $O\left(n^{2}\right)$ beams, the complexity is therefore $O(N n \log n)$ or $O\left(N n^{2}\right)$.

The most expensive part of the EGB algorithm is the evolution step. However, we can treat this as a pre-processing step since all the information can be re-used if we compute another solution 


\begin{tabular}{||c||c|c|c||}
\hline \hline & $\mathrm{AD}$ & $\mathrm{PM}$ & FBI \\
\hline Wave function Decomposition & $n$ & $n^{3}$ & $\beta$ \\
Evolution & $n^{3}$ & $n^{3}$ & $n^{3}$ \\
Extraction & $n \log n$ & $n \log n$ & - \\
Summation & $N n \log n$ & $N n \log n$ & $N n^{2}$ \\
\hline Total & $n^{3}+N n \log n$ & $n^{3}+N n \log n$ & $n^{3}+\beta+N n^{2}$ \\
\hline Each Extra Wave function & $N n \log n$ & $n^{3}+N n \log n$ & $\beta+N n^{2}$ \\
\hline \hline
\end{tabular}

Table 1: Complexity of various Eulerian Gaussian Beam algorithms.

\begin{tabular}{||c||c||}
\hline \hline FDCN & $s N^{2}$ \\
SP2 & $s N^{2} \log N$ \\
\hline LGB-AD & $s\left(n^{2}+N n\right)$ \\
LGB-PM & $s\left(n^{3}+N n\right)$ \\
LGB-FBI & $s\left(n^{3}+\beta+N n^{2}\right)$ \\
\hline EGB-AD & $n^{3}+s N n \log n$ \\
EGB-PM & $s n^{3}+s N n \log n$ \\
EGB-FBI & $n^{3}+s \beta+s N n^{2}$ \\
\hline \hline
\end{tabular}

Table 2: Complexity of various algorithms for solving $s$ different initial wave functions or different $\hbar$. 
with a different initial wave function or a different $\hbar$ under the same potential. To each of such extra initial wave functions, the complexity for finding the solution to the Schrödinger equation depends only on the initial wave function decomposition, zero level set extraction (if necessary) and a summation process. Let $s$ be the total number of different initial wave functions under the same potential. In Table 2, we compare the overall complexity of various algorithms. FDCN represents the finite difference Crank-Nicolson method and SP2 corresponds to the Strang Splitting Spectral method; see [2]. Even though the computational complexity of FDCN is slightly smaller than that of SP2, SP2 uses $N=O(1 / \hbar)$ grid points while FDCN requires $N=O\left(1 / \hbar^{1.5}\right)$ points to obtain a physically correct solution.

\section{$5 \quad$ Numerical Examples}

We show some numerical examples to validate our algorithms. Since the wave function is an auxiliary quantity used to compute primarily physical observables, such as the position density,

$$
I(x, t)=|U(x, t)|^{2},
$$

and the current density,

$$
J(x, t)=\hbar \operatorname{Im}[\overline{U(x, t)} \nabla U(x, t)]
$$

we will use these two quantities to justify our algorithms.

If an exact wave function for the Schrödinger equation is available, then we will use the resulting position and current densities to justify our beam-based position and current densities. If an exact wave function for the Schrödinger equation is not available, then we will use a direct numerical method to solve the Schrödinger equation first to obtain an "exact" wave function and use the resulting position and current densities to calibrate our beam solutions.

Here we will solve the Schrödinger equation by either the Crank-Nicolson scheme[2]

$$
\begin{aligned}
\frac{1}{\Delta t}\left(U_{j}^{n+1}-U_{j}^{n}\right)= & \frac{i \hbar}{4 \Delta x^{2}}\left(U_{j-1}^{n+1}-2 U_{j}^{n+1}+U_{j+1}^{n+1}+U_{j-1}^{n}-2 U_{j}^{n}+U_{j+1}^{n}\right) \\
& -\frac{i}{2 \hbar} V_{j}\left(U_{j}^{n+1}+U_{j}^{n}\right),
\end{aligned}
$$

where $U_{j}^{n}=U\left(x_{j}, t^{n}\right)$ and $V_{j}=V\left(x_{j}\right)$, or the following Strang Splitting Spectral Method (SP2) [2]

$$
\begin{aligned}
U_{j}^{*} & =\exp \left[-i V_{j} k / 2 \hbar\right] U_{j}^{n} \\
\hat{U}_{l}^{*} & =\sum_{j=0}^{M-1} U_{j}^{*} \exp \left[-i \mu_{l}\left(x_{j}-x_{\min }\right)\right] \\
U_{j}^{* *} & =\frac{1}{M} \sum_{l=-M / 2}^{M / 2} \exp \left[-i \hbar k \mu_{l}^{2} / 2\right] \hat{U}_{l}^{*} \exp \left[i \mu_{l}\left(x_{j}-x_{\min }\right)\right] \\
U_{j}^{n+1} & =\exp \left[-i V_{j} k / 2 \hbar\right] U_{j}^{* *},
\end{aligned}
$$

where $\mu_{l}=2 \pi l /\left(x_{\max }-x_{\min }\right)$ and $j=0, \cdots, N$. For a detailed analysis of these two finite difference methods, we refer readers to [2]. 


\subsection{Example 1: Free-Space Gaussian with focusing}

In this example, the initial wave function is in the form of a Gaussian with a nonlinear quadratic phase, given by

$$
U(x, t=0)=\frac{1}{\sqrt{\sigma_{0} \sqrt{2 \pi}}} \exp \left(-\frac{x^{2}}{4 \sigma_{0}^{2}}\right) \exp \left(-\frac{i x^{2}}{2 \hbar}\right),
$$

where $\sigma_{0}=0.1$ and $\hbar=2 \pi / 1024$.

We have plotted the ray trajectories in Figure 3(a) for beam evolution initialized by the asymptotic decomposition or the pointwise matching decomposition; all rays converge and form a caustic at $t=1$. When we look at the terminal locations of the bicharacteristics in the $x-p$ space at $t=0.5$ (Figure 3(b)), $t=1.0$ (Figure 3(c)), and $t=2.0$ (Figure 3(d)) we can see that the $p$-component of the terminal location as a function of the $x$-component changes from a single-valued function to a multi-valued function to a single-valued function.

Concerning the initial wave function decomposed using the FBI transform, we have shown the magnitude of the function $A_{0}(x, p)=\mathcal{T} U_{0}$ in Figure 4. Unlike the Fourier transform where the coefficients spread all over the phase space, the FBI transform provides a microlocalized representation in phase space. Since in this case we only shoot out beams if $\left|A_{0}\right|$ is non-zero, the number of beams we use to represent the initial wave function is much less than that in the case of using the Fourier transform.

To validate our algorithms in treating caustics, we compare the beam solutions with the finitedifference solution in terms of the position density as shown in Figures 5 to 9 . In the beam computation we discretize the $x-p$ space by a uniform $257 \times 257$ mesh and initialize the beams by four different strategies: (a) the asymptotic decomposition with $\epsilon=1$, (b) the asymptotic decomposition with $\epsilon=\hbar / \Delta x^{2}$, (c) the pointwise matching decomposition with $\epsilon=\hbar / \Delta x^{2}$, and (d) the FBI transform with $\epsilon=1$. The finite-difference solution is obtained by directly applying the Crank-Nicolson scheme to the Schrödinger equation with a mesh of $N=2^{15}$ and $\Delta t=10^{-4}$. Since the solution is smooth before and after the caustic, the differences between the finite-difference position density and the beam-based position density are not visible in Figures 5 and 7 . If, however, we zoom in the peak regions, then we will see the differences as shown in Figures 6 and 8 . At $t=1$, we have a caustic, and Figure 9 shows the beam solutions based on different initialization strategies.

\subsection{Example 2: Simple Harmonic Oscillator}

The simple harmonic oscillator has an external potential,

$$
V(x)=\frac{1}{2} \omega^{2} x^{2},
$$

where $\omega$ is the potential's natural angular frequency of oscillation. Consider the following onedimensional wave packet

$$
U(x, t=0)=\left(\frac{\omega}{\pi \hbar}\right)^{1 / 4} \exp \left(-\frac{\omega(x-\bar{x})^{2}}{2 \hbar}\right) \exp \left(\frac{i \bar{p} x}{\hbar}\right) .
$$

This is a minimum wave packet [25] (page 446): it represents a particle with unit mass localized in configuration space about its mean position $\bar{x}$ with a root-mean-square deviation $\Delta x=\sqrt{\hbar / 2 \omega}$ 
(a)
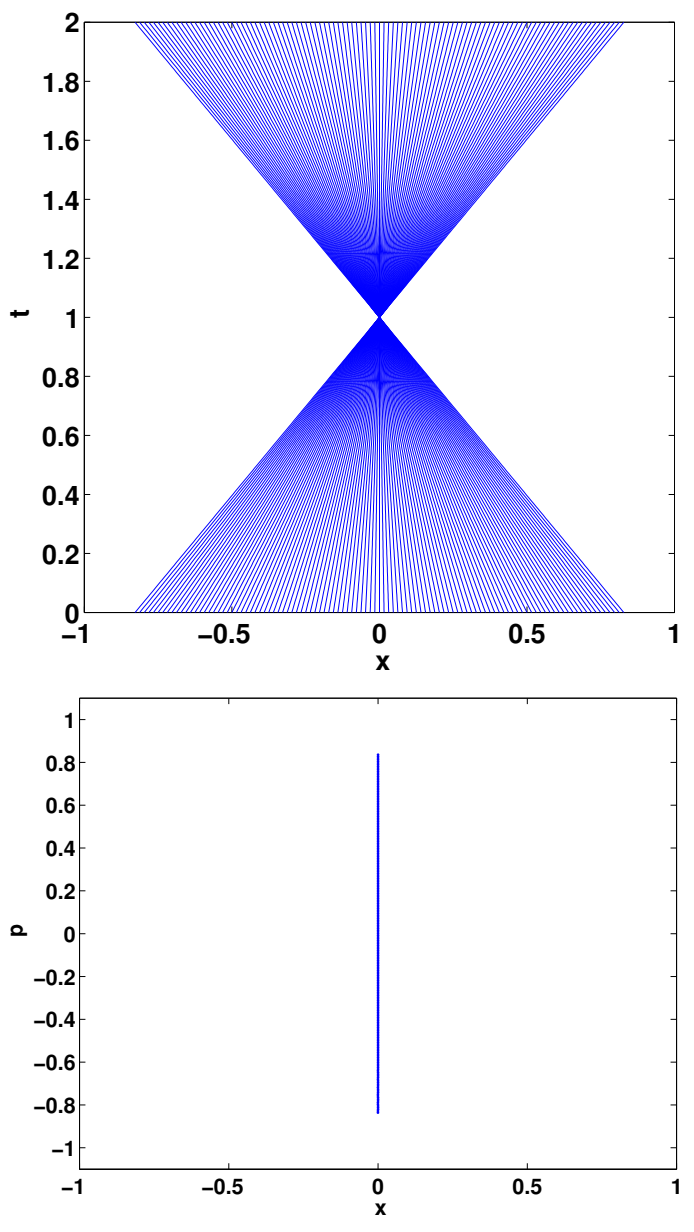

(b)

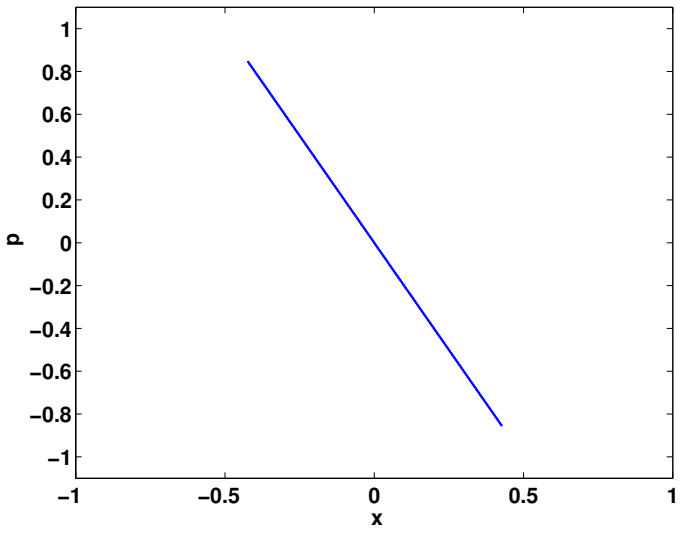

(d)

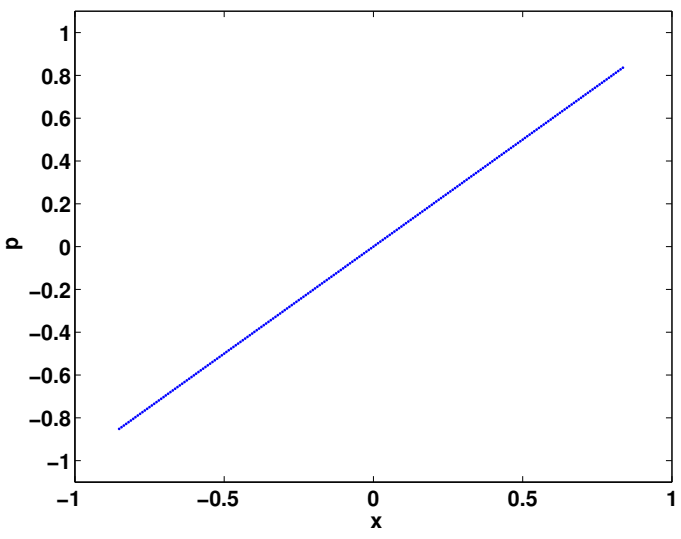

Figure 3: (Example 5.1) The initial wave function is decomposed using the asymptotic decompositions or the pointwise matching decomposition. (a) Rays in the $x$ - $t$ space. (b) The terminal locations of bicharacteristics in the $x-p$ space at $t=0.5$. (c) The terminal locations of bicharacteristics in the $x-p$ space at $t=1.0$. (d) The terminal locations of bicharacteristics in the $x$ - $p$ space at $t=2.0$. 


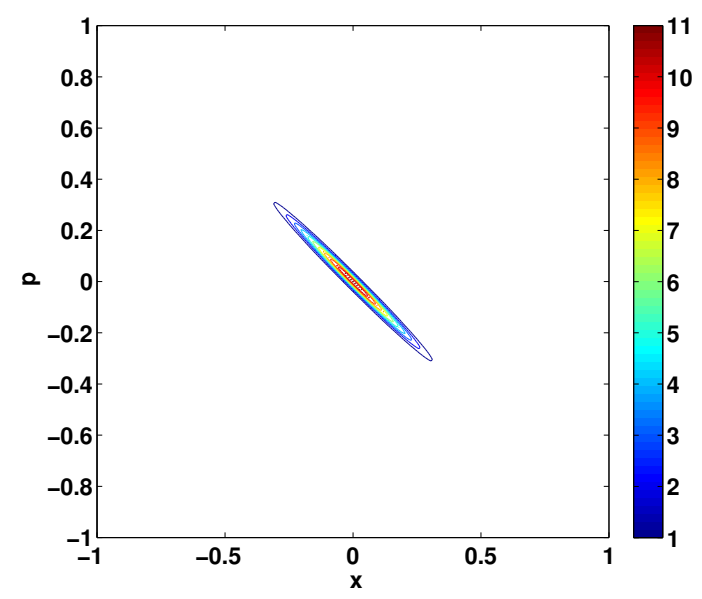

Figure 4: (Example 5.1) Contour plot of $\left|A_{0}\right|$ obtained by embedding the initial wave function in the phase space using the FBI transform.

and localized in momentum space about its mean position $\bar{p}$ with a root-mean-square deviation $\Delta p=\sqrt{\hbar \omega / 2}$.

When this particle is subject to the harmonic oscillation, it can be shown that such a packet retains minimum size in the course of time and that it oscillates with a frequency $\omega$. Moreover, the wave packet evolves according to the following form

$$
U(x, t)=\left(\frac{\omega}{\pi \hbar}\right)^{1 / 4} \exp \left(-\frac{\omega\left(x-\bar{x}_{t}\right)^{2}}{2 \hbar}\right) \exp \left(\frac{i \bar{p}_{t} x}{\hbar}\right)
$$

where $\bar{p}_{t}$ ad $\bar{x}_{t}$ are the mean values of $p$ and $x$ at time $t$. Here we remark that $\bar{p}_{t}$ and $\bar{x}_{t}$ are given by the classical trajectory (or the central ray) in the beam construction. Moreover, the current density is given by

$$
I(x, t)=\left(\frac{\omega}{\pi \hbar}\right)^{1 / 2} \exp \left(-\frac{\omega\left(x-\bar{x}_{t}\right)^{2}}{\hbar}\right) .
$$

We have computed the evolution of a Gaussian packet for $t=2$ with $\hbar=0.01$ and $\bar{p}=-0.5$ under a harmonic potential of $\omega=2$. Figure 10 shows the errors in position densities computed by the Eulerian Gaussian beams according to different initialization strategies.

\subsection{Example 3: smooth solution with a constant potential}

This example is taken from [20,2]. The background potential is taken to be $V(x)=100$ and the initial condition is given by

$$
U(x, t=0)=\exp \left[-25(x-0.5)^{2}\right] \exp \left[\frac{i \tilde{\tau}_{0}(x)}{\hbar}\right]
$$

where

$$
\tilde{\tau}_{0}(x)=0.2\left(x^{2}-x\right) .
$$


(a)
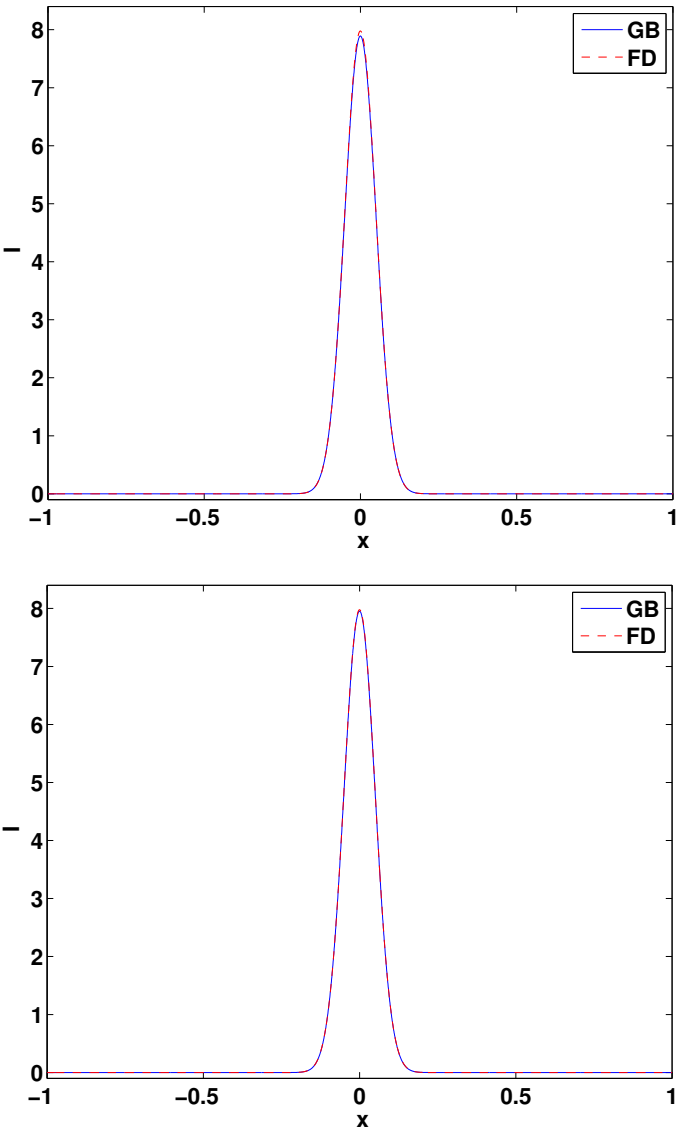

(b)
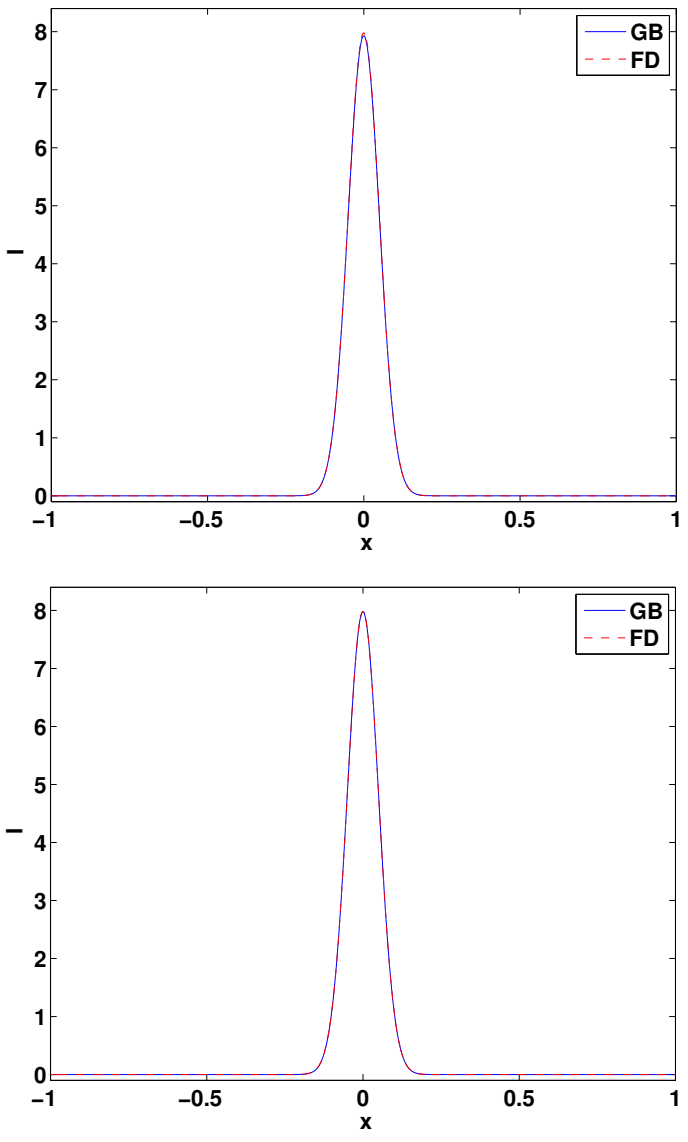

Figure 5: (Example 5.1) Comparison of the position density at $t=0.5$ (before caustic) computed by the Crank-Nicolson scheme and Eulerian Gaussian beams for $h=1 / 1024$. The Crank-Nicolson position density is computed with a mesh $N=2^{15}$ and $\Delta t=10^{-4}$. (a) The asymptotic decomposition with $\epsilon=1$, (b) the asymptotic decomposition with $\epsilon=\hbar / \Delta x^{2}$, (c) the pointwise matching decomposition with $\epsilon=\hbar / \Delta x^{2}$, and (d) the FBI transform with $\epsilon=1$. 
(a)
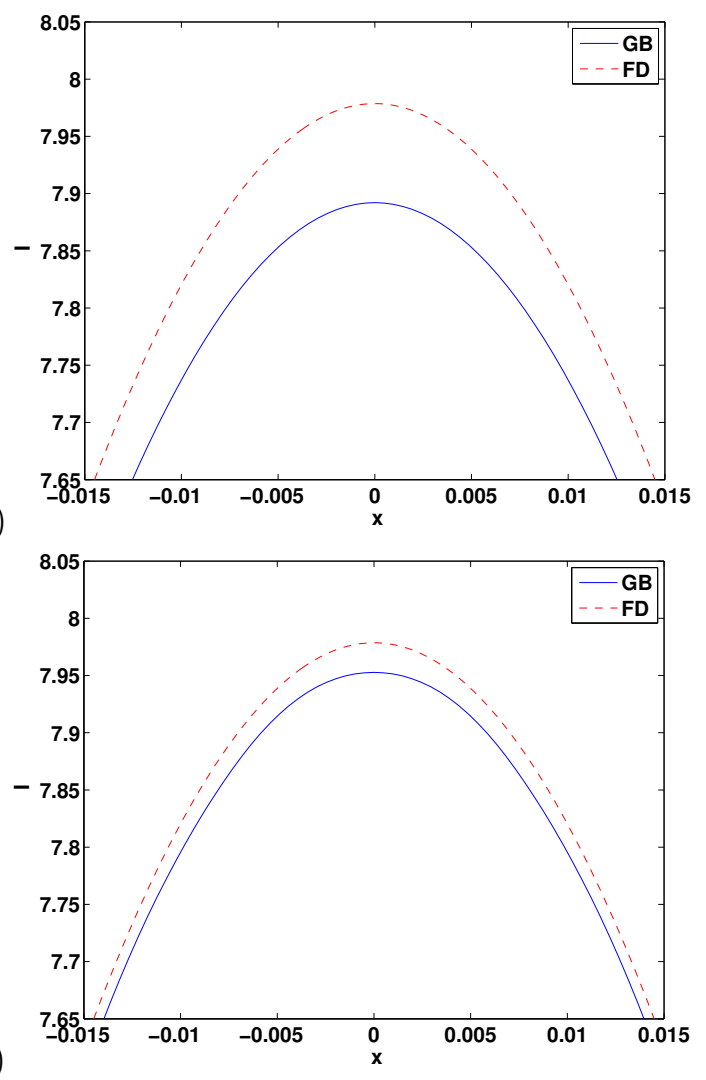

(b)
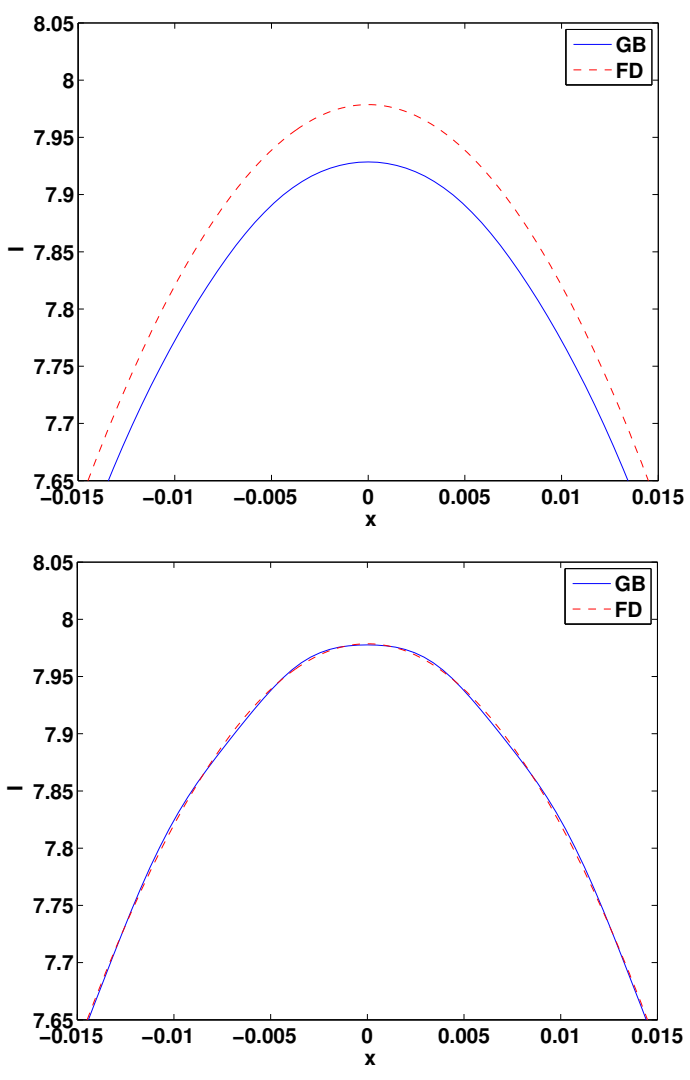

Figure 6: (Example 5.1) A zoom in of figure 5. Comparison of the position density at $t=0.5$ (before caustic) computed by the Crank-Nicolson scheme and Eulerian Gaussian beams for $h=1 / 1024$. The Crank-Nicolson position density is computed with a mesh $N=2^{15}$ and $\Delta t=10^{-4}$. (a) The asymptotic decomposition with $\epsilon=1$, (b) the asymptotic decomposition with $\epsilon=\hbar / \Delta x^{2}$, (c) the pointwise matching decomposition with $\epsilon=\hbar / \Delta x^{2}$, and (d) the FBI transform with $\epsilon=1$. 
(a)
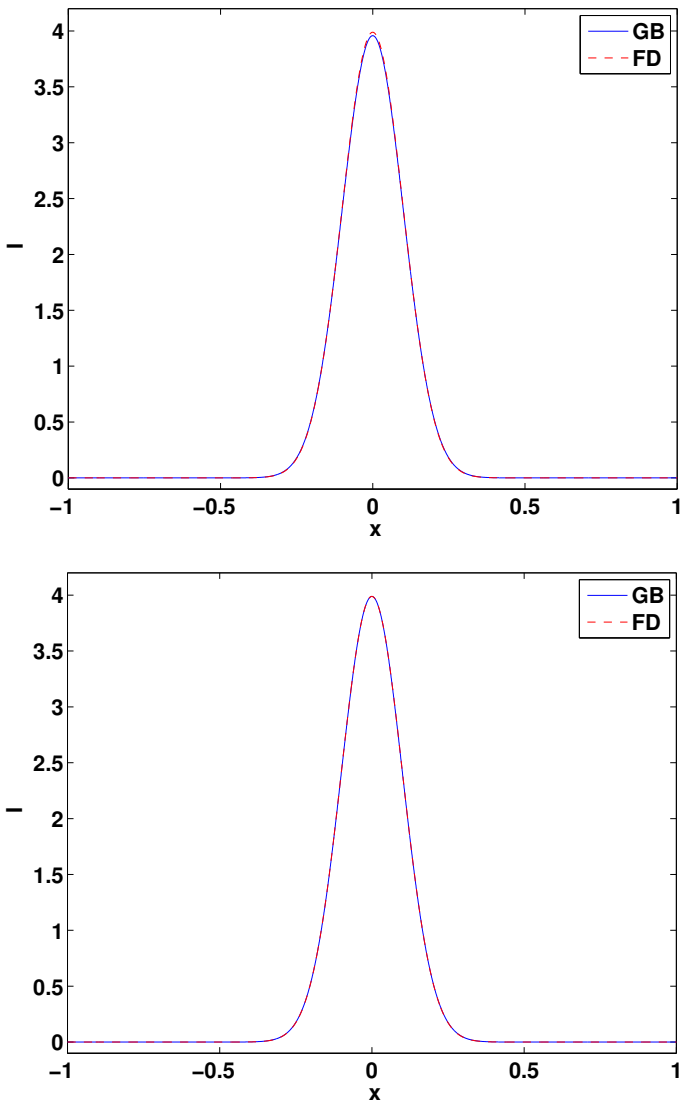

(b)
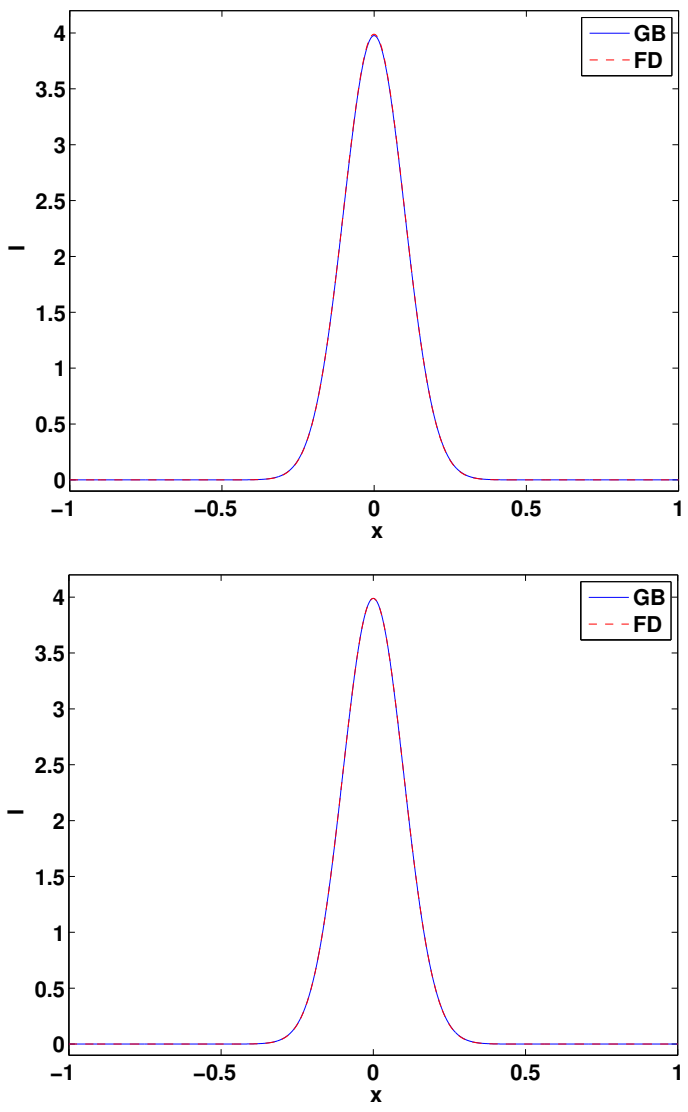

Figure 7: (Example 5.1) Comparison of the position density at $t=2.0$ (after caustic) computed by the Crank-Nicolson scheme and Eulerian Gaussian beams for $h=1 / 1024$. The Crank-Nicolson position density is computed with a mesh $N=2^{15}$ and $\Delta t=10^{-4}$. (a) The asymptotic decomposition with $\epsilon=1$, (b) the asymptotic decomposition with $\epsilon=\hbar / \Delta x^{2}$, (c) the pointwise matching decomposition with $\epsilon=\hbar / \Delta x^{2}$, and (d) the FBI transform with $\epsilon=1$. 
(a)
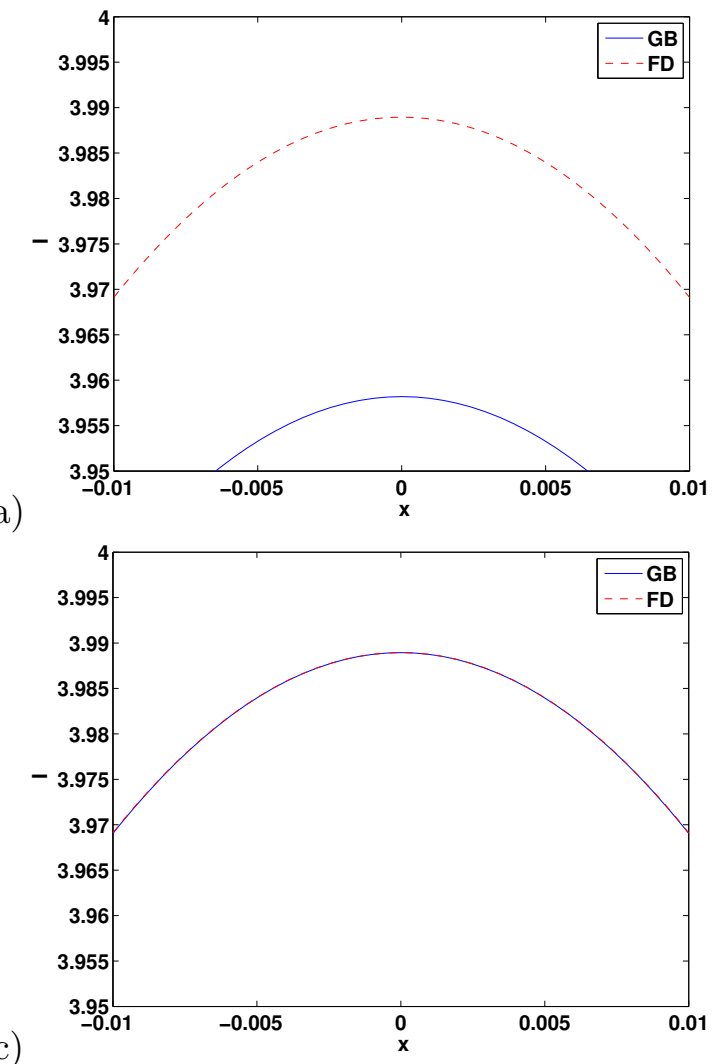

(b)

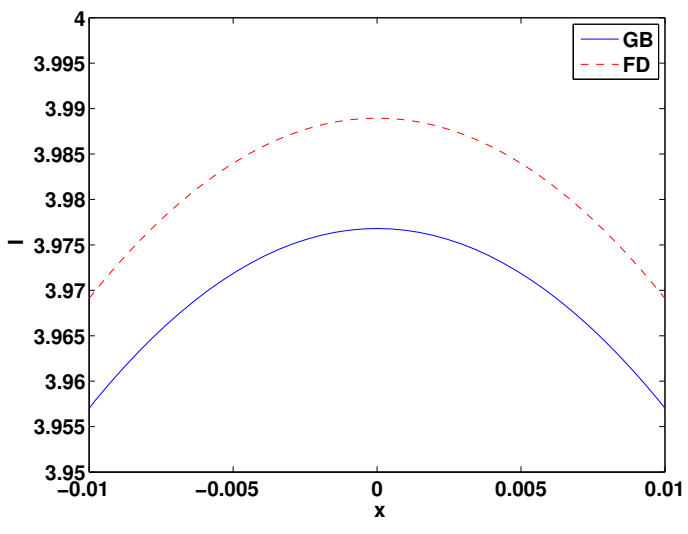

(d)

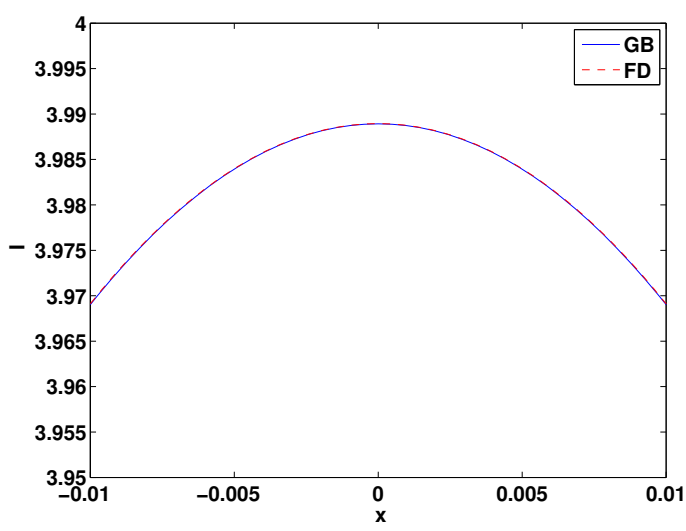

Figure 8: (Example 5.1) A zoom in of figure 7. Comparison of the position density at $t=2.0$ (before caustic) computed by the Crank-Nicolson scheme and Eulerian Gaussian beams for $h=1 / 1024$. The Crank-Nicolson position density is computed with a mesh $N=2^{15}$ and $\Delta t=10^{-4}$. (a) The asymptotic decomposition with $\epsilon=1$, (b) the asymptotic decomposition with $\epsilon=\hbar / \Delta x^{2}$, (c) the pointwise matching decomposition with $\epsilon=\hbar / \Delta x^{2}$, and (d) the FBI transform with $\epsilon=1$. 
(a)
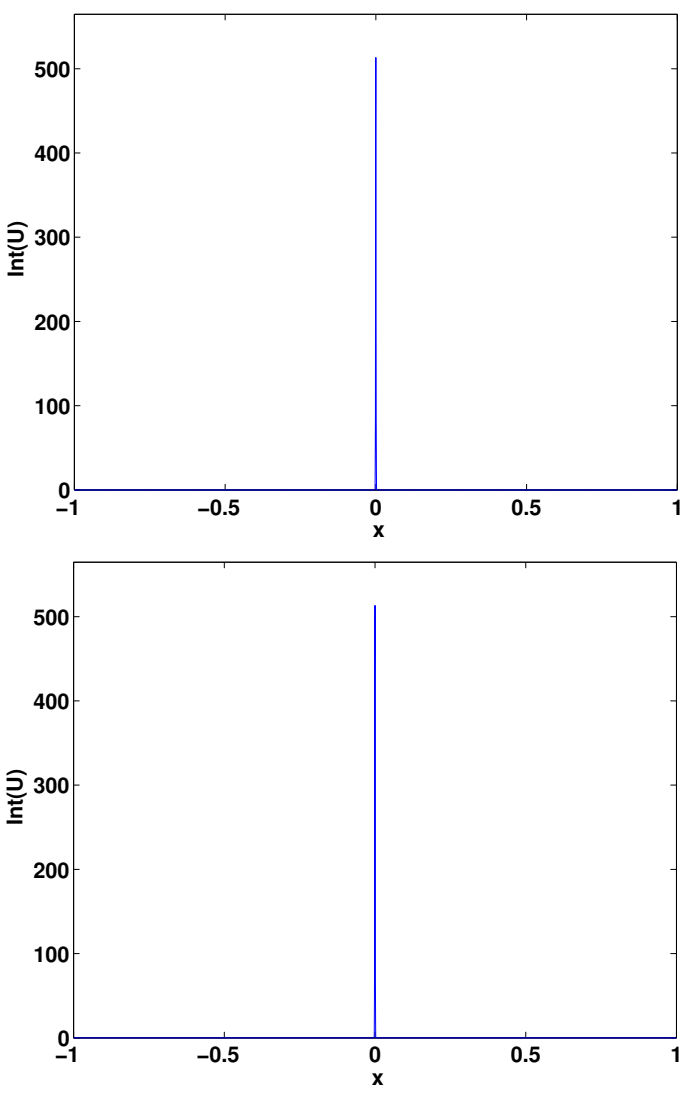

(b)

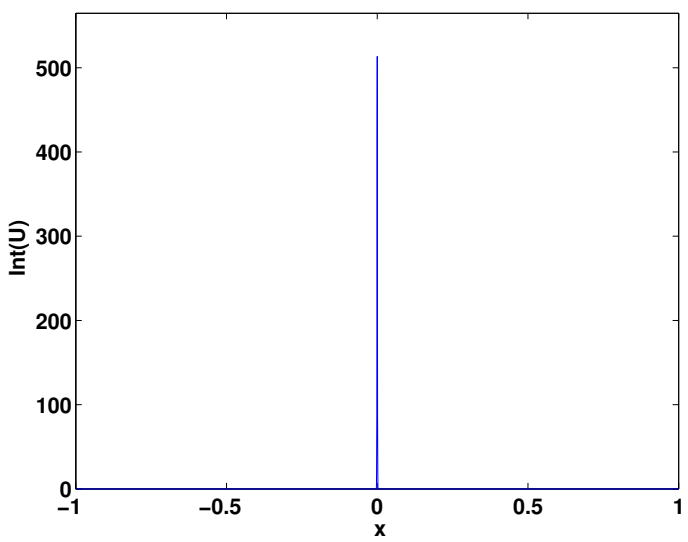

(d)

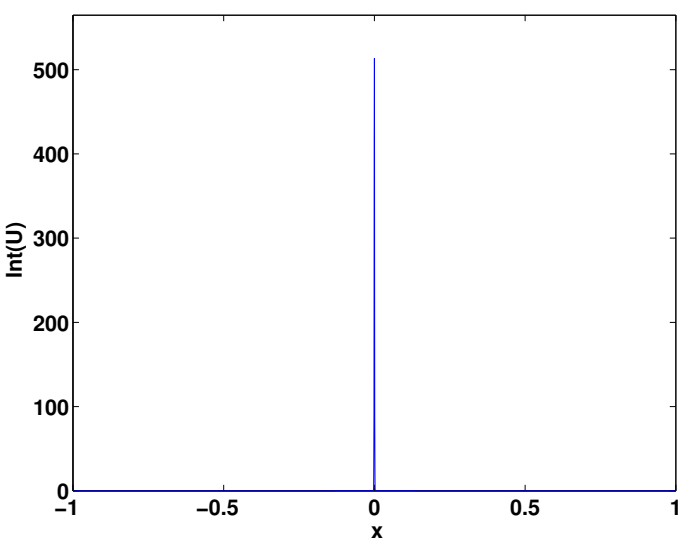

Figure 9: (Example 5.1) The position density at $t=1.0$ (at caustic) for $h=1 / 1024$ with the initial wave function decomposed using (a) the asymptotic decomposition with $\epsilon=1$, (b) the asymptotic decomposition with $\epsilon=\hbar / \Delta x^{2}$, (c) the pointwise matching decomposition with $\epsilon=\hbar / \Delta x^{2}$, and (d) the FBI transform with $\epsilon=1$. 
(a)
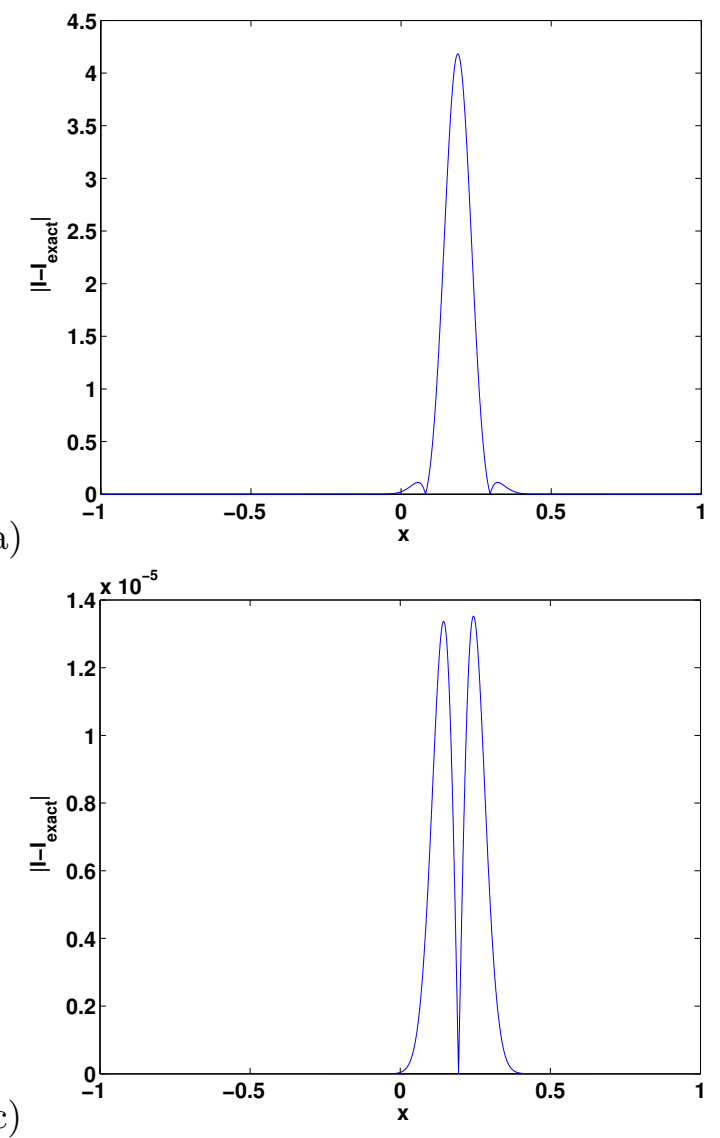

(b)
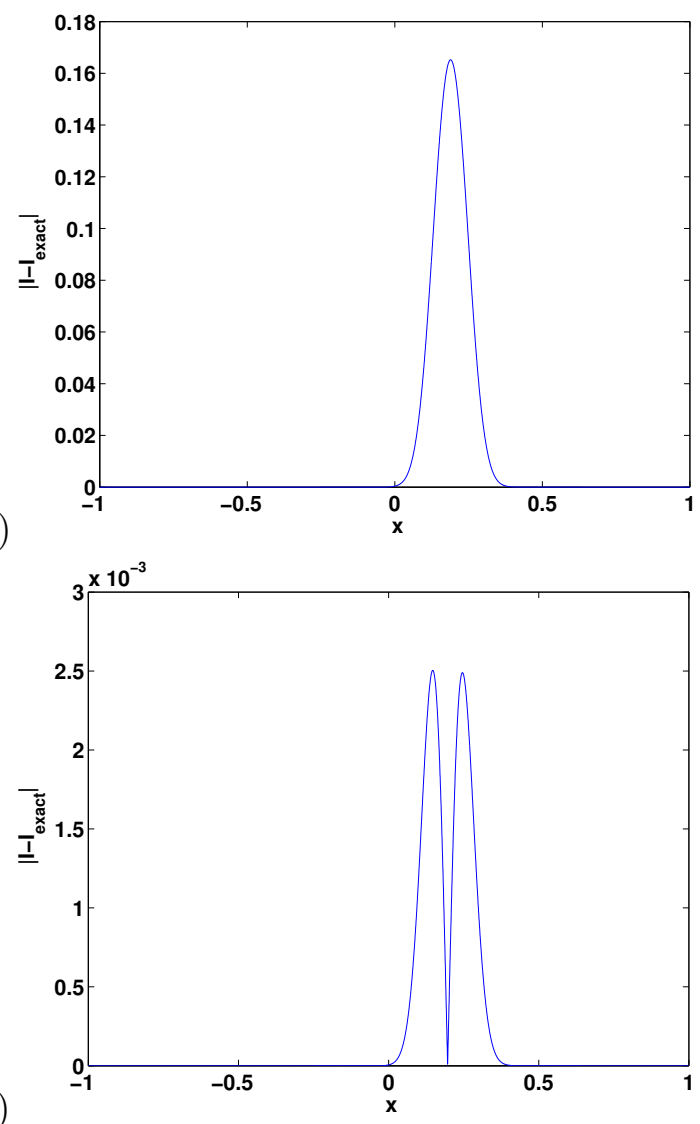

Figure 10: (Example 5.2) The errors in the position density using Eulerian Gaussian beams with 129 grid points in each direction of the phase space. The initial wave function is decomposed using (a) the asymptotic decomposition with $\epsilon=1$, (b) the asymptotic decomposition with $\epsilon=\hbar / \Delta x^{2}$, (c) the pointwise matching decomposition $\epsilon=\hbar / \Delta x^{2}$, and (d) the FBI transform with $\epsilon=1$. 
We compare the beam based solutions with the Crank-Nicolson finite-difference solutions at $t=0.54$. In terms of beams we use a uniform mesh of $257 \times 257$ grid points to discretize the $x$ - $p$ space. In terms of the Crank-Nicolson finite-difference scheme we use a uniform mesh of $N=24576$ and a time step of $\Delta t=10^{-5}$. We illustrate the behavior of our algorithms as $h$ tends to zero.

Figure 11 shows the comparison of position densities computed by the two methods, where the beam solutions are based on the asymptotic decomposition with $\epsilon=1$ and $\epsilon=\hbar / \Delta x^{2}$. As we can see in the figure, the asymptotic decomposition with $\epsilon=\hbar / \Delta x^{2}$ is more accurate than that with $\epsilon=1$. Although the beam solution is Figure 11 (f) has some tiny oscillations, which are due to insufficient number of beams, those oscillations can be removed if we increase the number of beams as will be shown in a late example.

Figure 12 shows the comparison of position densities computed by the two methods, where the beam solutions are based on the pointwise matching decomposition with $\epsilon=\hbar / \Delta x^{2}$ or the FBI based decomposition with $\epsilon=1$. Overall the FBI based beam summation is more accurate than the pointwise matching decomposition based beam summation since the former uses much more beams than the latter.

\subsection{Example 4: smooth solution with a quadratic potential}

We borrow another example from [2]. The initial wave function has the same form as equation (94) with a linear initial phase function $\tilde{\tau}_{0}(x)=x+1$. The external potential is harmonic given by $V(x)=x^{2} / 2$.

We compare the beam based solutions with the time-splitting spectral finite-difference solutions (SP2) at $t=0.54$. In terms of beams we use a uniform mesh of $257 \times 257$ grid points to discretize the $x-p$ space. In terms of the time-splitting spectral finite-difference scheme we use a uniform mesh of $N=1024$ and a time step of $\Delta t=10^{-2}$. We illustrate the behavior of our algorithms for $\hbar=1 / 400$ and $\hbar=1 / 6400$, respectively.

Figure 13 compares the solutions $\hbar=1 / 400$ obtained by the SP2 scheme and the beam solutions based on four different approaches to initialize the beams. As we can see, the FBI based beam solution has the best behavior among the four beam solutions.

Figure 14 compares the solutions $\hbar=1 / 6400$ obtained by the SP2 scheme and the beam solutions based on four different approaches to initialize the beams. As we can see, although all the four beam solutions approximate the "true" solution reasonably well, there are some tiny oscillations. However, those oscillations are gone if we increase the number of beams as shown in Figure 15.

\subsection{Example 5: caustics}

This example is also taken from [20,2]. The background potential is given by $V(x)=10$ and the initial wave function has the same form as equation (94) with the initial phase function $\tilde{\tau}_{0}(x)$ given by

$$
\tilde{\tau}_{0}(x)=-\frac{1}{5} \ln [\exp (5(x-0.5))+\exp (-5(x-0.5))] .
$$

As illustrated in $[20,2]$, caustics will form in this example as shown in Figure 16. For example, when initializing beams based on the asymptotic decomposition, initially only one ray departs from 
(a)
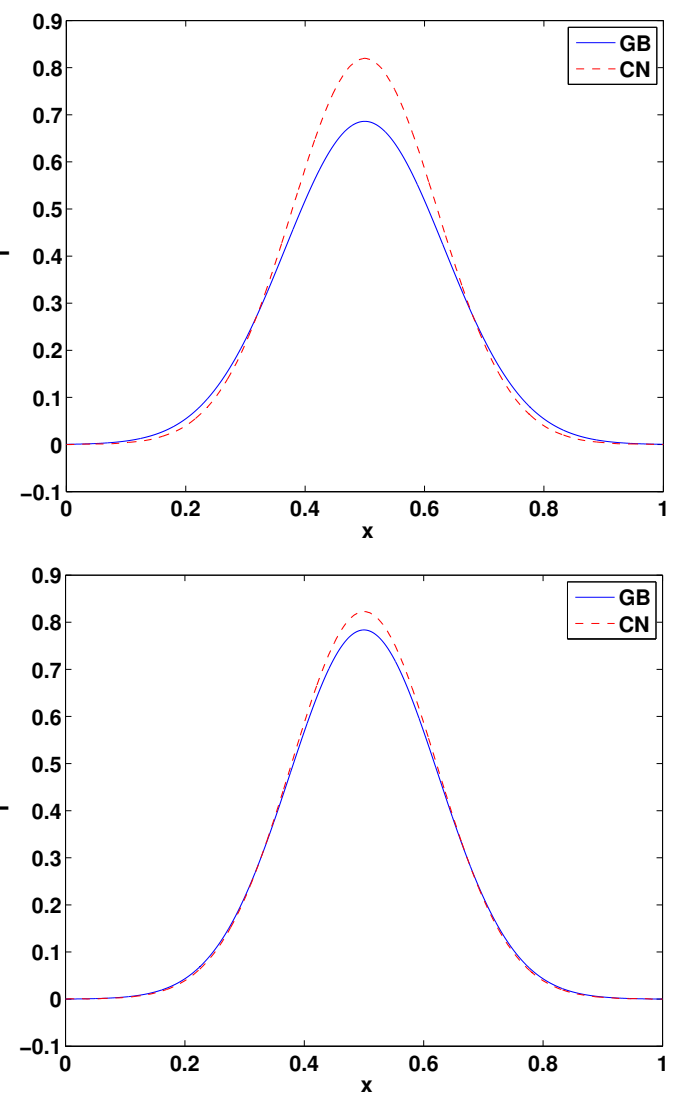

(c)

(e)

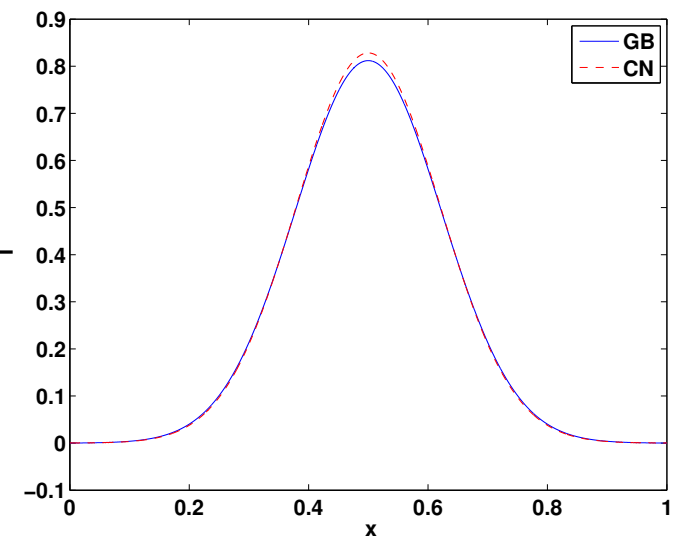

(b)
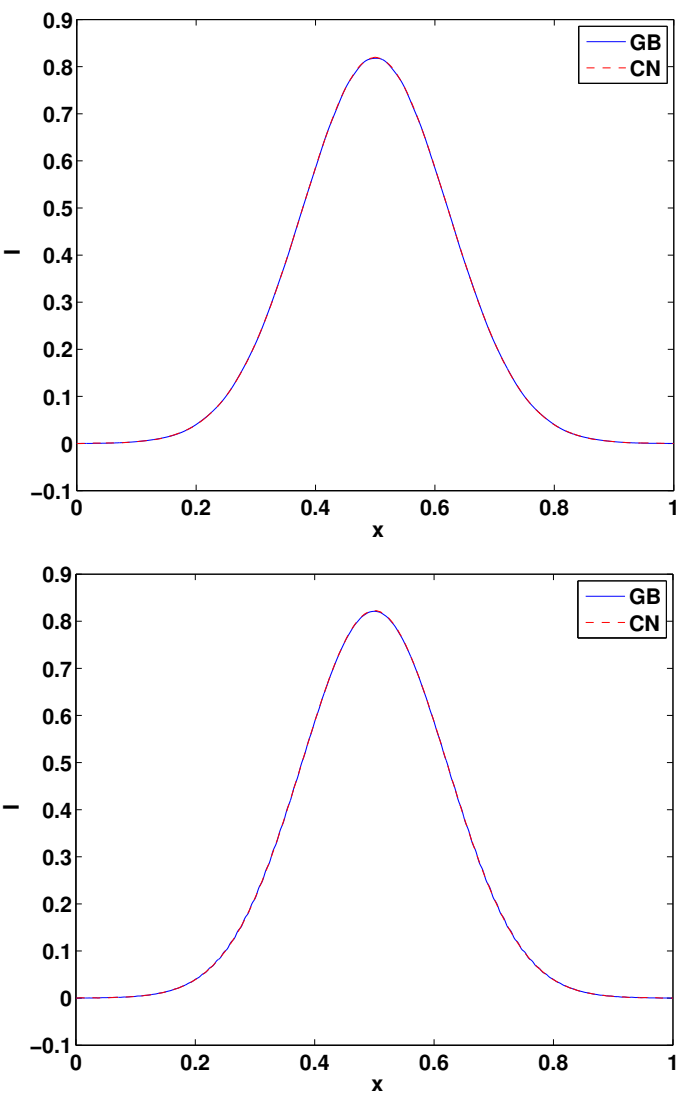

(d)

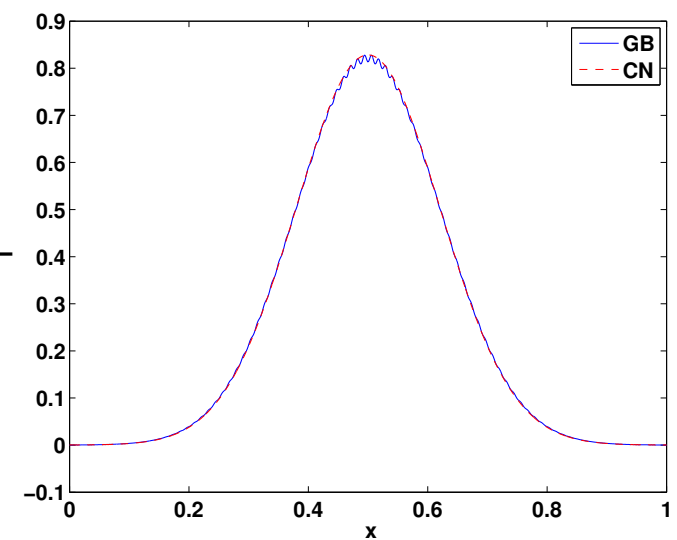

Figure 11: (Example 5.3) Comparison of the position densities computed by the Crank-Nicolson scheme and Eulerian Gaussian beams based on the asymptotic decomposition for the initial condition. The Crank-Nicolson position density is computed with a mesh $N=24576$ and $\Delta t=10^{-5}$. (a) $\epsilon=1$ and $\hbar=1 / 256$, (b) $\epsilon=\hbar / \Delta x^{2}$ and $\hbar=1 / 256$, (c) $\epsilon=1$ and $\hbar=1 / 1024$, (d) $\epsilon=\hbar / \Delta x^{2}$ and $\hbar=1 / 1024$, (e) $\epsilon=1$ and $\hbar=1 / 4096$, and (f) $\epsilon=\hbar / \Delta x^{2}$ and $\hbar=1 / 4096$. 
(a)

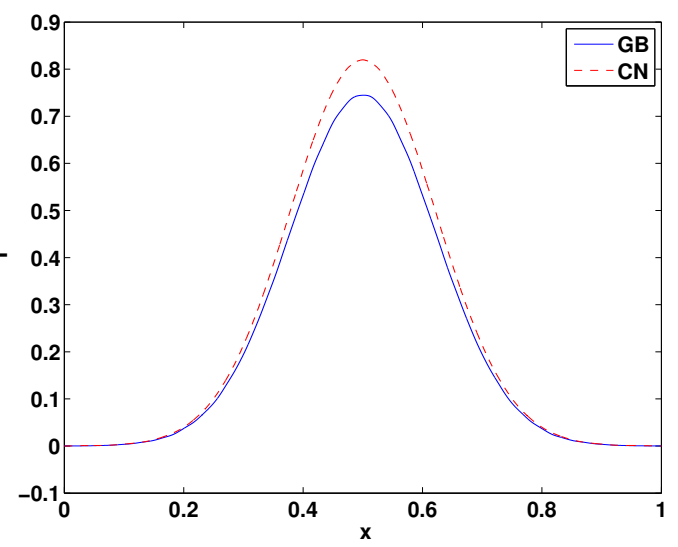

(c)

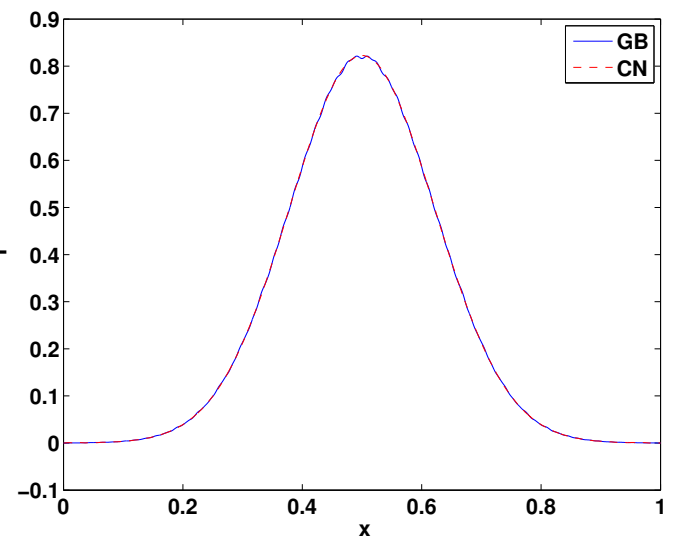

(d)

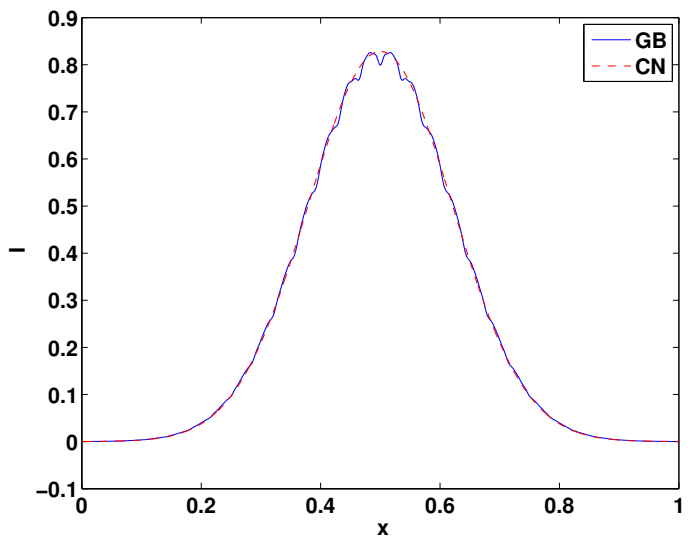

(b)
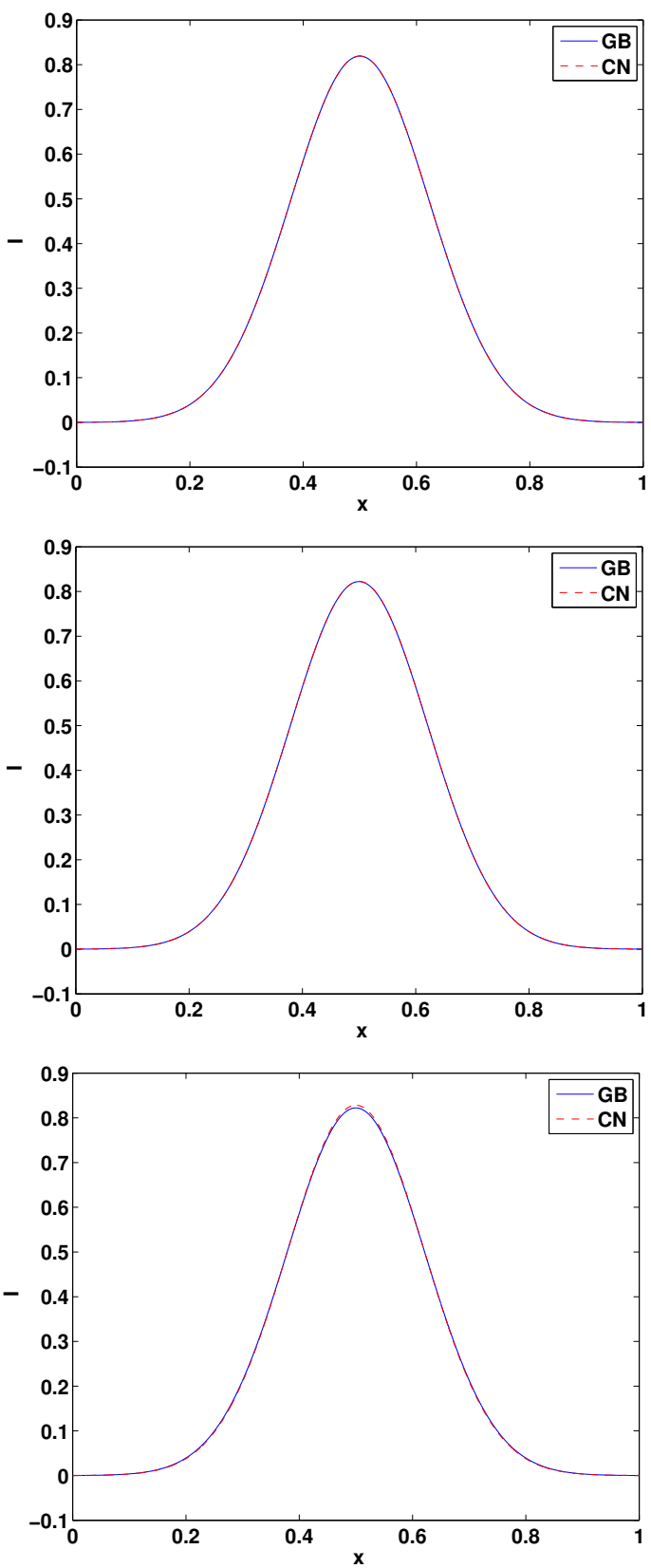

Figure 12: (Example 5.3) Comparison of the position densities computed by Eulerian Gaussian beams and the Crank-Nicolson scheme. The Crank-Nicolson position density is computed with a mesh $N=24576$ and $\Delta t=10^{-5}$. (a) The pointwise matching decomposition with $\epsilon=\hbar / \Delta x^{2}$ and $\hbar=1 / 256$, (b) the FBI transform based decomposition with $\epsilon=1$ and $\hbar=1 / 256$, (c) the pointwise matching decomposition with $\epsilon=\hbar / \Delta x^{2}$ and $\hbar=1 / 1024$, (d) the FBI transform based decomposition with $\epsilon=1$ and $\hbar=1 / 1024$, (e) the pointwise matching decomposition with $\epsilon=\hbar / \Delta x^{2}$ and $\hbar=1 / 4096$, and (f) the FBI transform based decomposition with $\epsilon=1$ and $\hbar=1 / 4096$. 
(a)

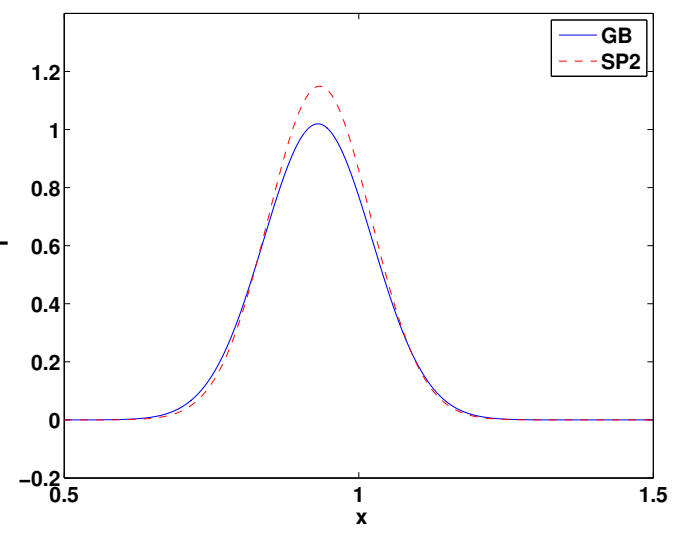

(c)

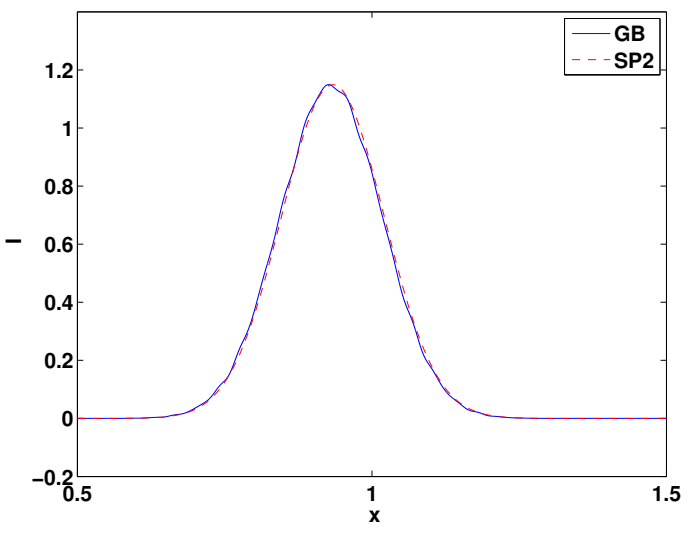

(b)

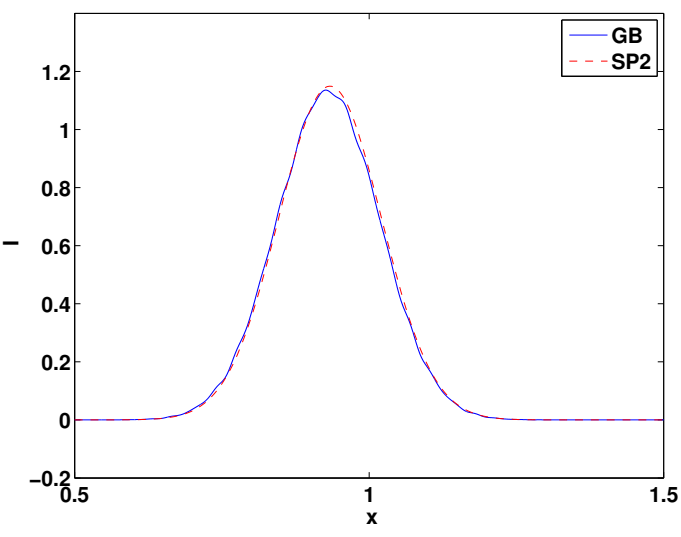

(d)

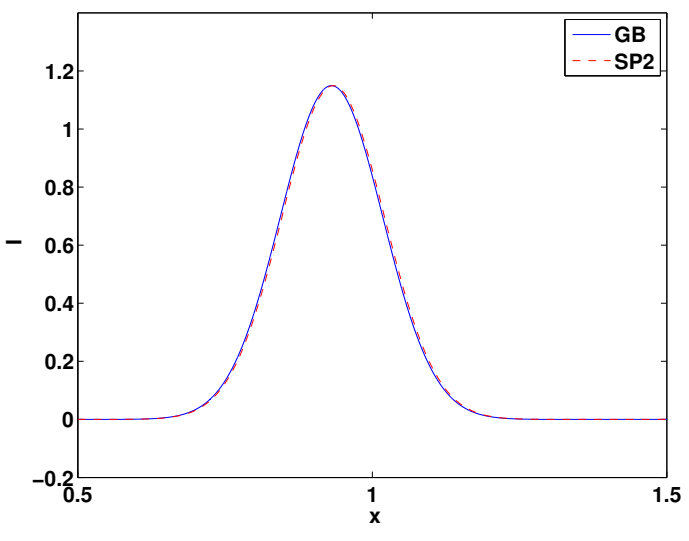

Figure 13: (Example 5.4) Comparison of the position densities computed by Eulerian Gaussian beams and the SP2 scheme for $\hbar=1 / 400$. The Gaussian-beam position density is computed with a uniform $257 \times 257$ mesh in the phase space. The SP2 position density is computed with a mesh $N=2^{10}$ and $\Delta t=2 \times 10^{-2}$. The initial condition is obtained by (a) the asymptotic decomposition with $\epsilon=1$, (b) the asymptotic decomposition with $\epsilon=\hbar / \Delta x^{2}$, (c) the pointwise matching decomposition with $\epsilon=\hbar / \Delta x^{2}$, and (d) the FBI transform with $\epsilon=1$. 
(a)

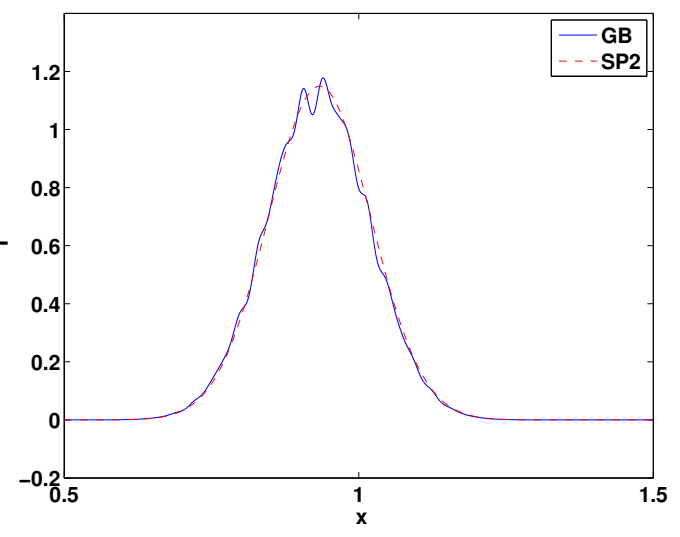

(c)

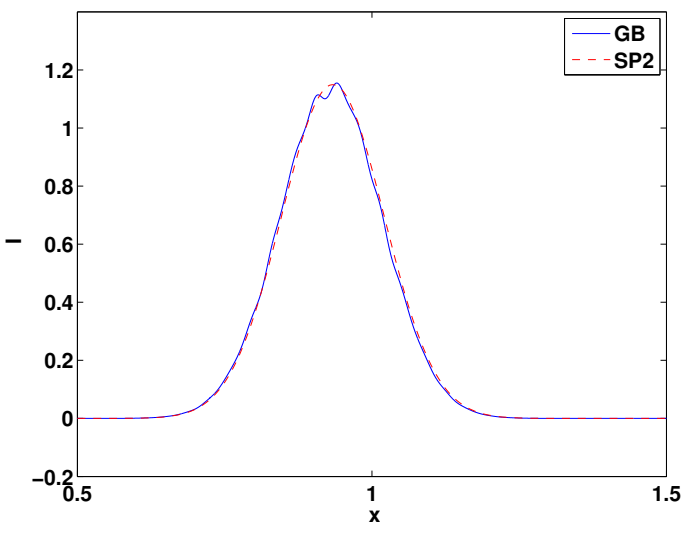

(b)

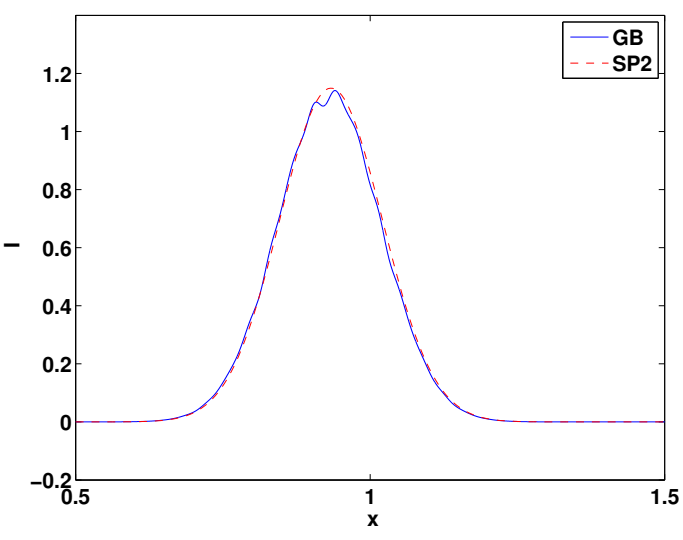

(d)

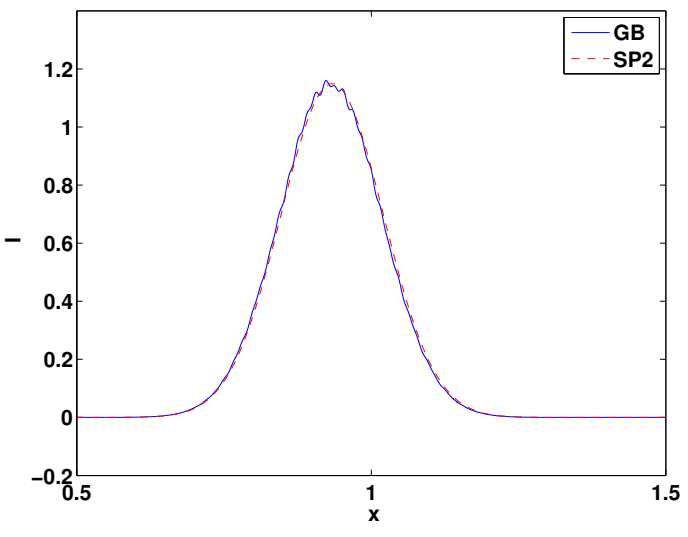

Figure 14: (Example 5.4) Comparison of the position densities computed by Eulerian Gaussian beams and the SP2 scheme for $\hbar=1 / 6400$. The Gaussian-beam position density is computed with a uniform $257 \times 257$ mesh in the phase space. The SP2 position density is computed with a mesh $N=2^{10}$ and $\Delta t=2 \times 10^{-2}$. The initial condition is obtained by (a) the asymptotic decomposition with $\epsilon=1$, (b) the asymptotic decomposition with $\epsilon=\hbar / \Delta x^{2}$, (c) the pointwise matching decomposition with $\epsilon=\hbar / \Delta x^{2}$, and (d) the FBI transform with $\epsilon=1$. 
(a)

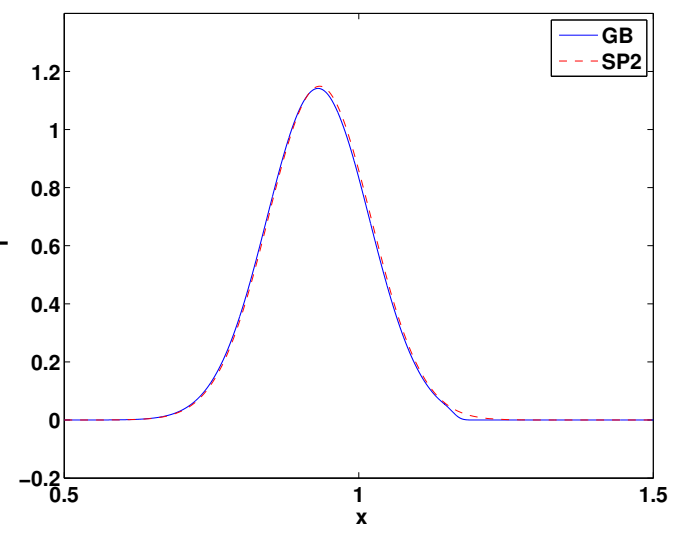

(c)

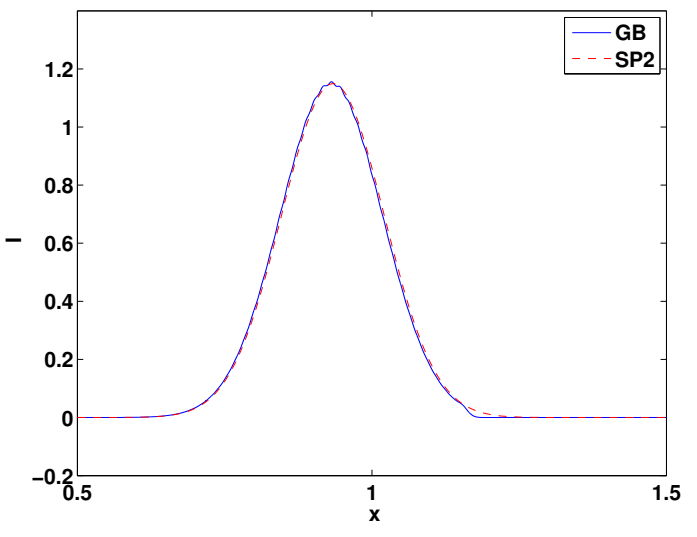

(b)

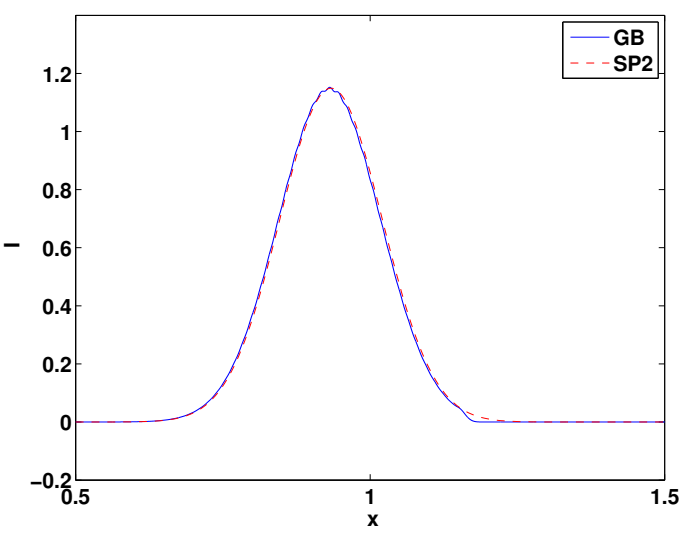

(d)

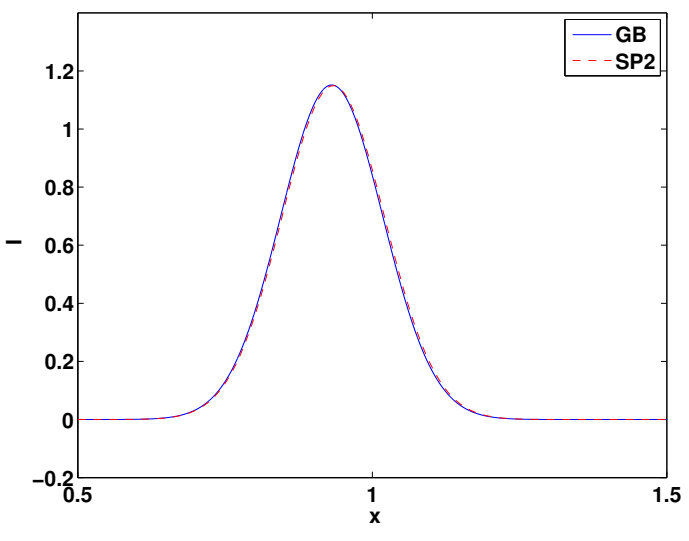

Figure 15: (Example 5.4) Comparison of the position densities computed by Eulerian Gaussian beams and the SP2 scheme for $\hbar=1 / 6400$. The Eulerian Gaussian-beam position density is computed with a uniform $513 \times 513$ mesh in the phase space. The SP2 position density is computed with a mesh $N=2^{10}$ and $\Delta t=2 \times 10^{-2}$. The initial condition is obtained by (a) the asymptotic decomposition with $\epsilon=1$, (b) the asymptotic decomposition with $\epsilon=\hbar / \Delta x^{2}$, (c) the pointwise matching decomposition with $\epsilon=\hbar / \Delta x^{2}$, and (d) the FBI transform with $\epsilon=1$. 
(a)

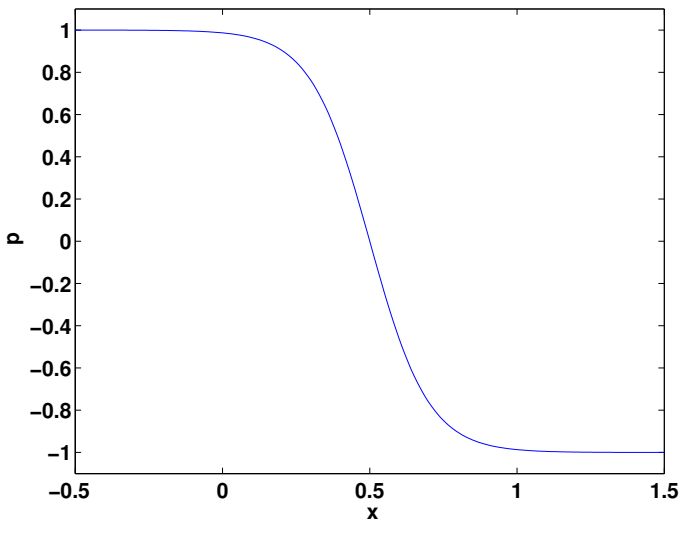

(b)

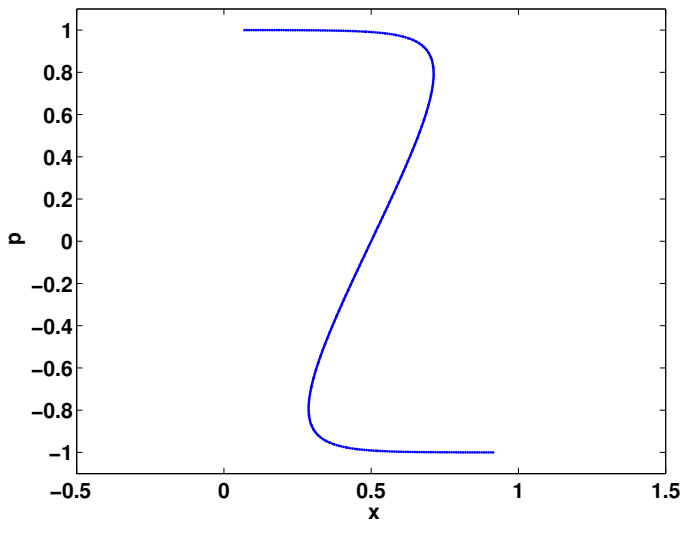

Figure 16: (Example 5.5) The initial wave function is decomposed using the asymptotic decompositions or the pointwise matching decomposition. (a) Initial locations of bicharacteristics in the $x-p$ space, and (b) terminal locations of bicharacteristics in the $x-p$ space.

each physical location $x$ with a corresponding ray parameter $p$ as illustrated in Figure 16(a), we plot the derivative of the phase function $\tilde{\tau}_{0}(x)$; however, at a later time there are three rays arriving at some physical locations $x$ with three different ray parameters $p$ as illustrated in Figure 16(b).

We compare the beam based solutions with the Strang Splitting Spectral solutions at $t=0.54$. In terms of beams we use a uniform mesh of $257 \times 257$ grid points to discretize the $x$ - $p$ space. In terms of the Strang Splitting Spectral scheme we use a uniform mesh of $N=32768$ and a time step of $\Delta t=10^{-5}$. We illustrate the behavior of our algorithms as $h$ tends to zero.

Since the initial condition is not represented well using the asymptotic decomposition with $\epsilon=1$, the solutions show large errors, as shown in Figure 17. Because the errors made in initializing the beams converges to zero in the order of $O(\hbar)$, the accuracy does improve as $\hbar$ decreases.

The solutions are significantly improved when initializing the beams using the asymptotic decomposition with $\epsilon=\hbar / \Delta x^{2}$, as shown in Figure 18. As $\hbar$ decreases, given the uniform mesh we will not have enough beams to resolve tiny scales of the oscillations; therefore, the errors for $\hbar=1 / 1024$ are larger than those in the other two cases $(\hbar=1 / 64$ or $\hbar=1 / 256)$.

Figures 19 and 20 compare the Strang Splitting Spectral solutions with the beam solutions based on the pointwise matching decomposition and the FBI decomposition, respectively. Overall the FBI based beam summation is more accurate than the pointwise matching decomposition based beam summation since the former uses much more beams than the latter.

\section{Conclusions}

We propose Gaussian-beam based Eulerian methods to compute semi-classical solutions of the Schrödinger equation. A new Eulerian framework uses global Cartesian coordinates, level-set based implicit representation and Liouville equations. The resulting method gives uniformly distributed phases and amplitudes in phase space simultaneously. To obtain semi-classical solutions to the Schrödinger equation with different initial wave functions, we only need to slightly modify the 
(a)
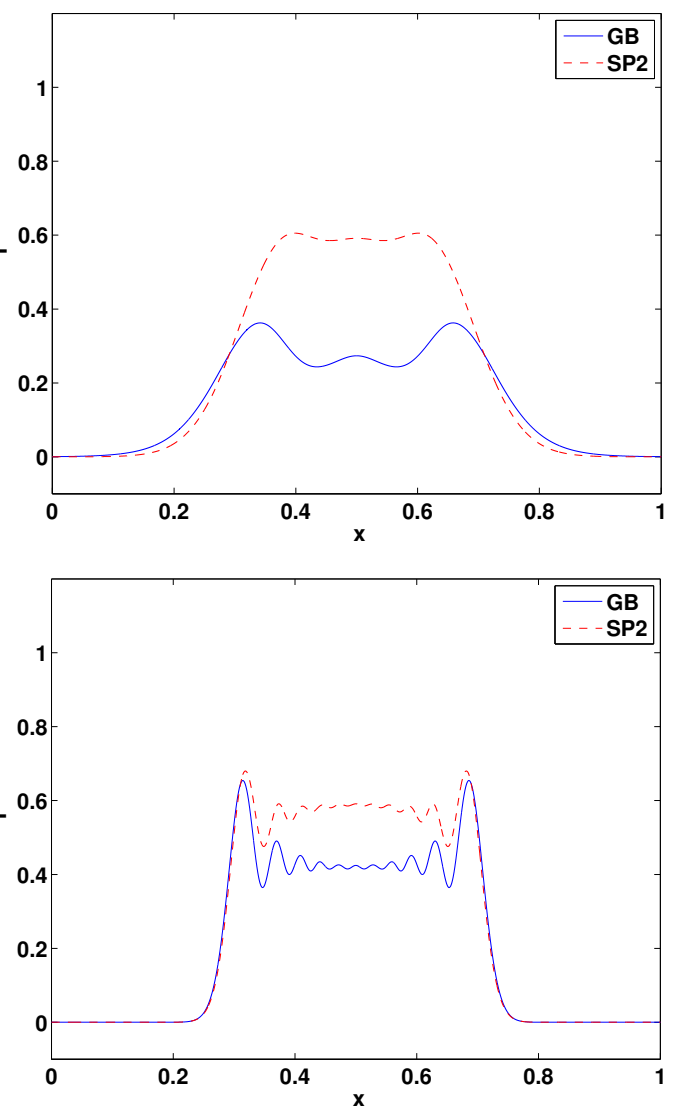

(c)

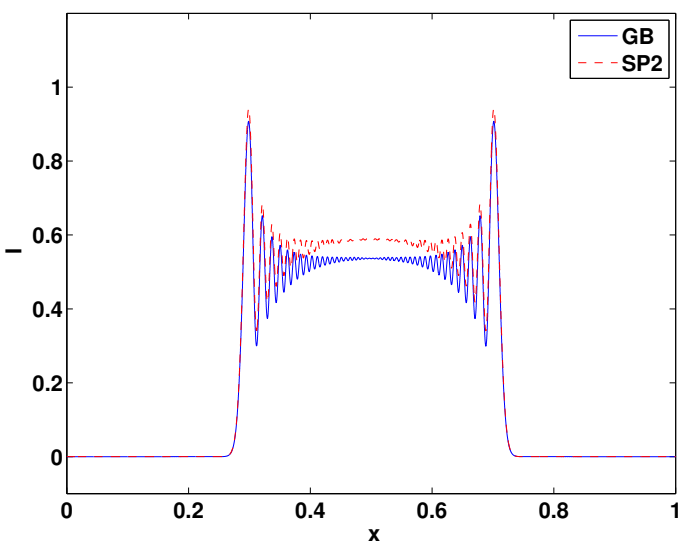

(b)

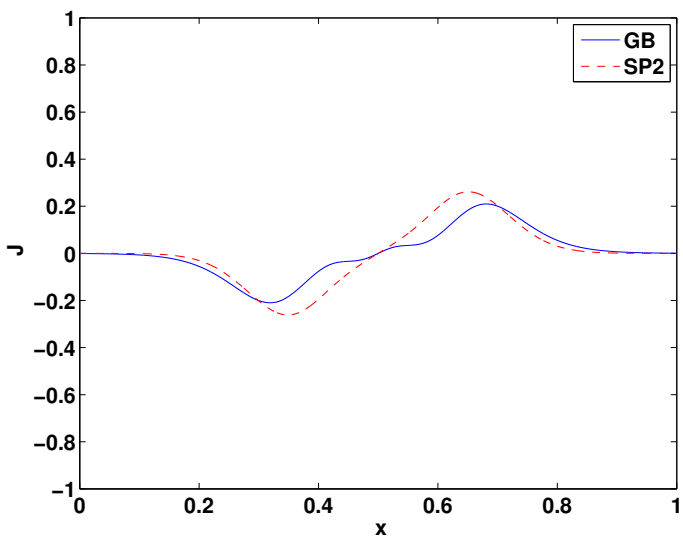

(d)
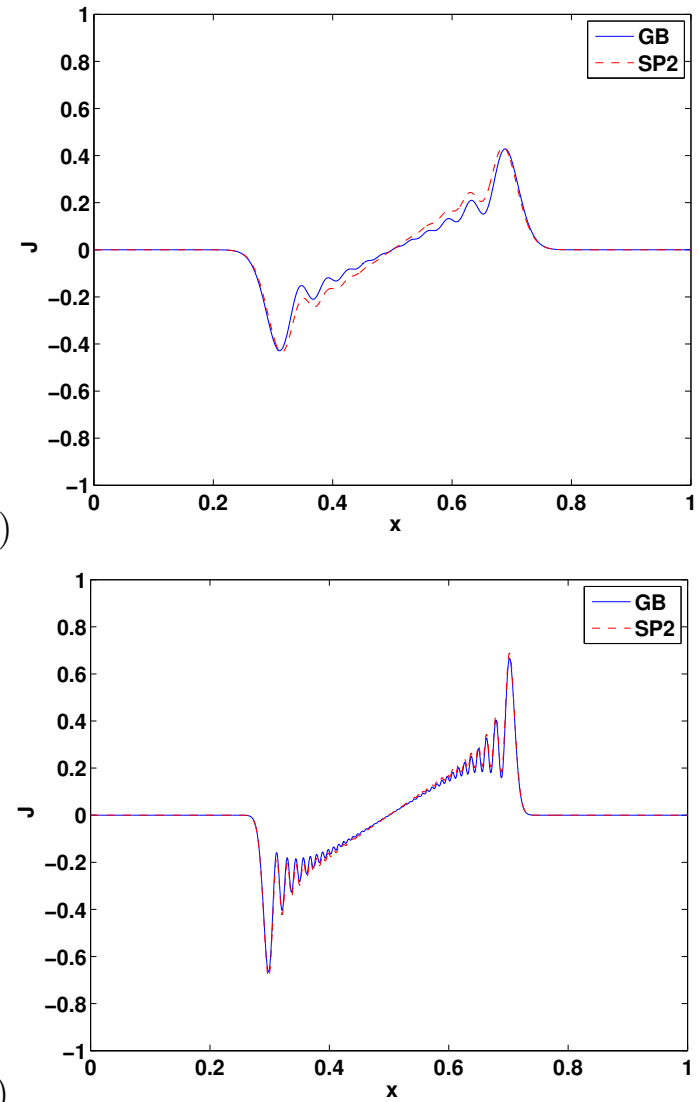

Figure 17: (Example 5.5) Comparison of the position densities computed by Eulerian Gaussian beams and the Strang Splitting Spectral scheme. The Eulerian Gaussian beam is based on the asymptotic decomposition for the initial condition with $\epsilon=1$. The Strang Splitting Spectral position density is computed with a mesh $N=2^{15}$ and $\Delta t=10^{-5}$. (a) The position densities with $\hbar=1 / 64$; (b) the current densities with $\hbar=1 / 64$; (c) the position densities with $\hbar=1 / 256$; (d) the current densities with $\hbar=1 / 256$; (e) the position densities with $\hbar=1 / 1024$; (f) the current densities with $\hbar=1 / 1024$. 
(a)

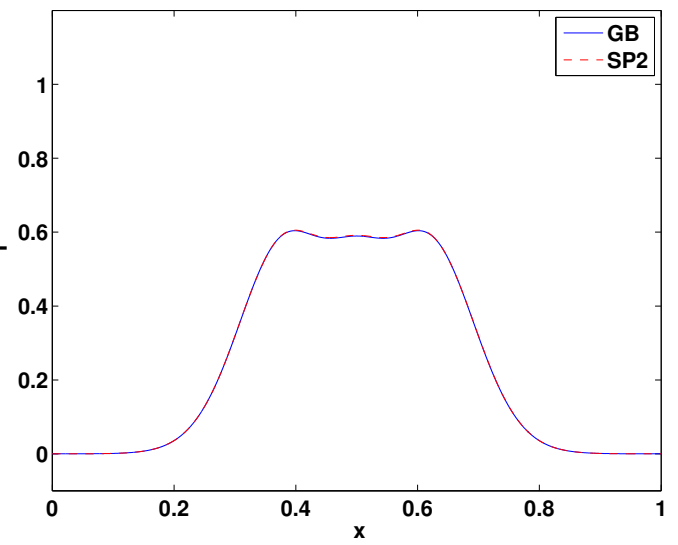

(c)

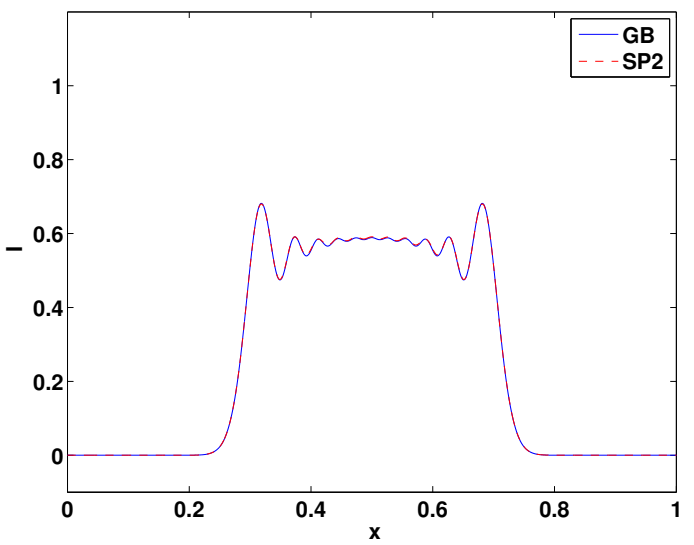

(d)

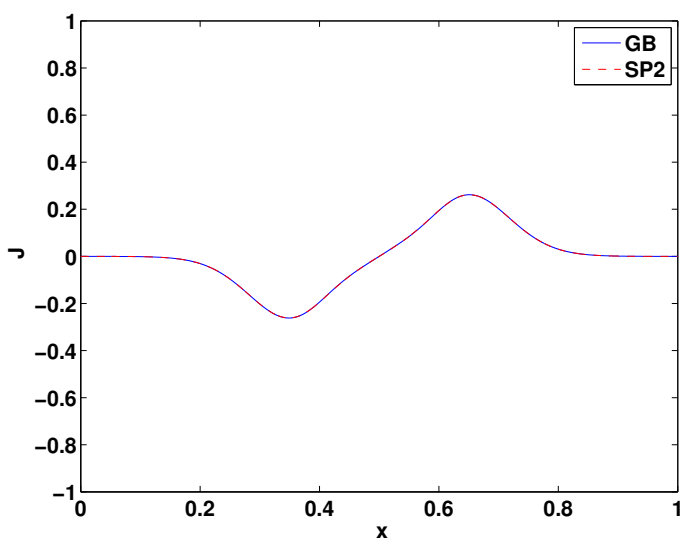

(b)
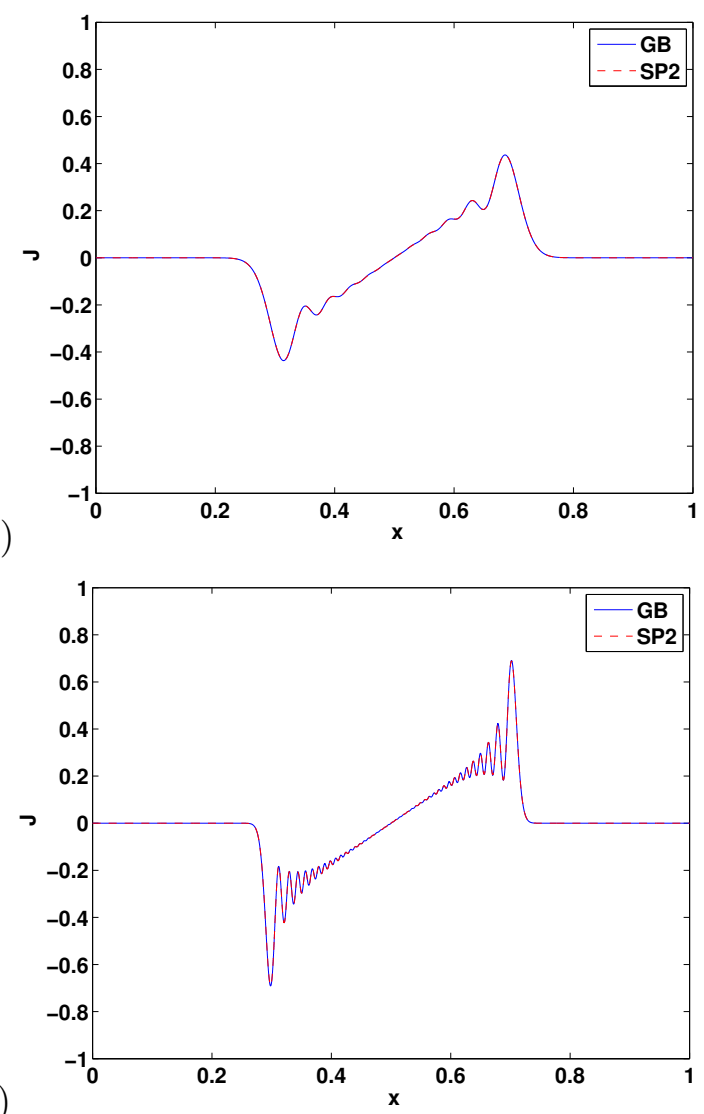

(e)

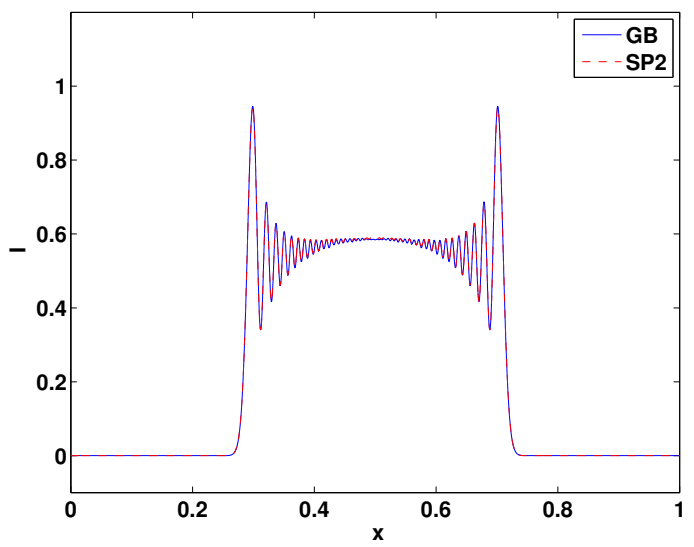

(f)

Figure 18: (Example 5.5) Comparison of the position densities computed by Eulerian Gaussian beams and the Strang Splitting Spectral scheme. The Eulerian Gaussian beam is based on the asymptotic decomposition for the initial condition with $\epsilon=\hbar / \Delta x^{2}$. The Strang Splitting Spectral position density is computed with a mesh $N=2^{15}$ and $\Delta t=10^{-5}$. (a) The position densities with $\hbar=1 / 64$; (b) the current densities with $\hbar=1 / 64$; (c) the position densities with $\hbar=1 / 256$; (d) the current densities with $\hbar=1 / 256$; (e) the position densities with $\hbar=1 / 1024$; (f) the current densities with $\hbar=1 / 1024$. 
(a)

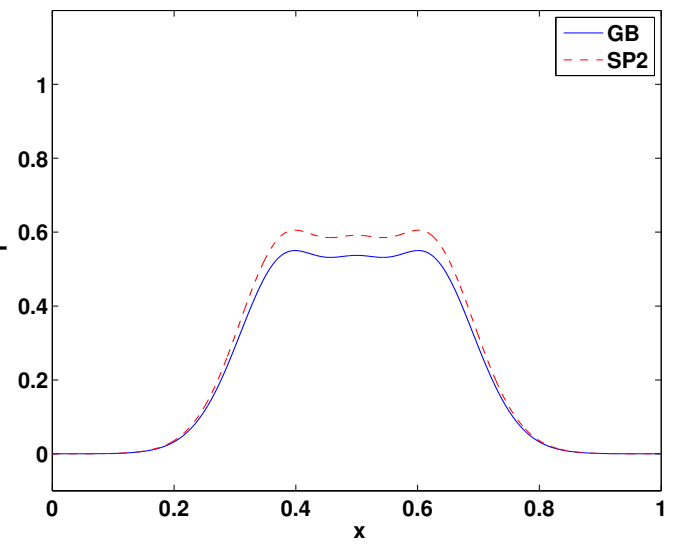

(c)

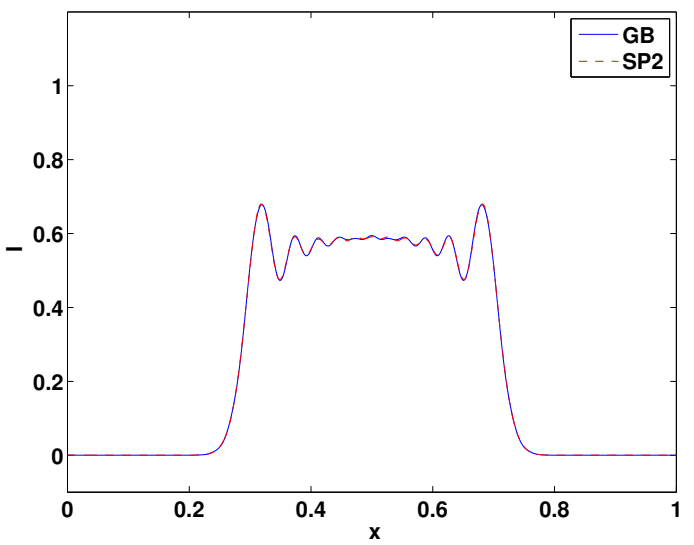

(d)

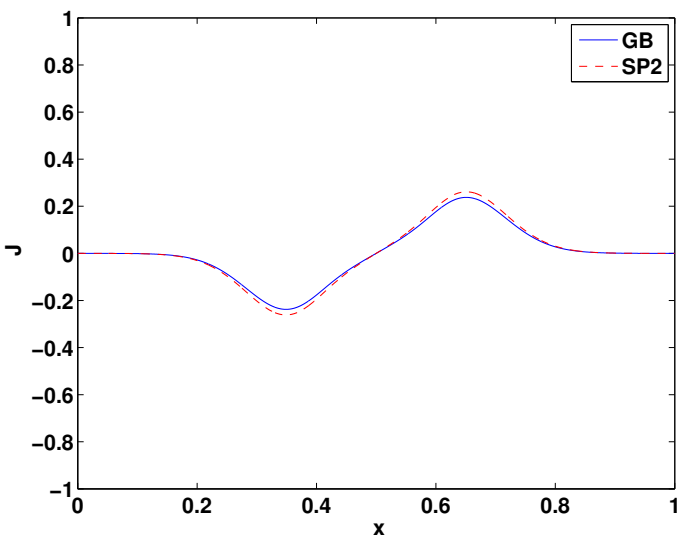

(b)
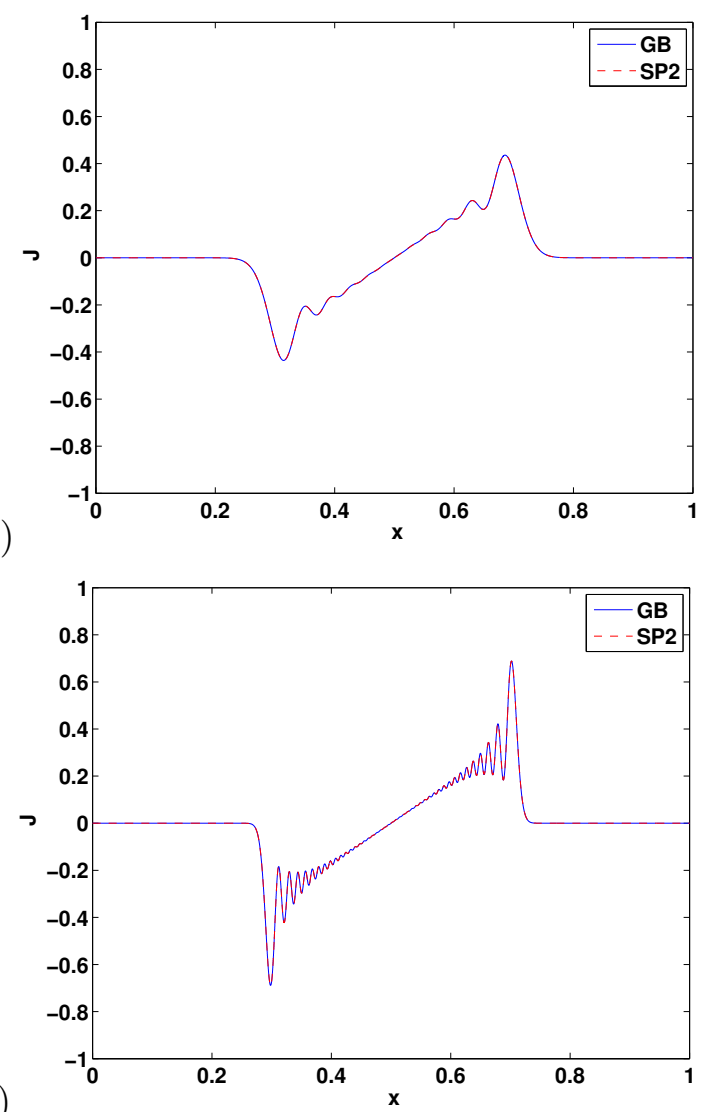

(e)

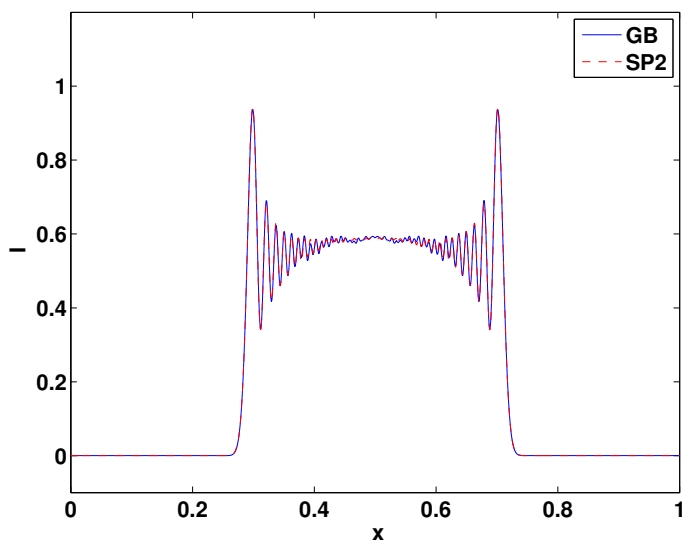

(f)

Figure 19: (Example 5.5) Comparison of the position densities computed by Eulerian Gaussian beams and the Strang Splitting Spectral scheme. The Eulerian Gaussian beam is based on the pointwise matching decomposition for the initial condition with $\epsilon=\hbar / \Delta x^{2}$. The Strang Splitting Spectral position density is computed with a mesh $N=2^{15}$ and $\Delta t=10^{-5}$. (a) The position densities with $\hbar=1 / 64$; (b) the current densities with $\hbar=1 / 64$; (c) the position densities with $\hbar=1 / 256$; (d) the current densities with $\hbar=1 / 256$; (e) the position densities with $\hbar=1 / 1024$; (f) the current densities with $\hbar=1 / 1024$. 
(a)
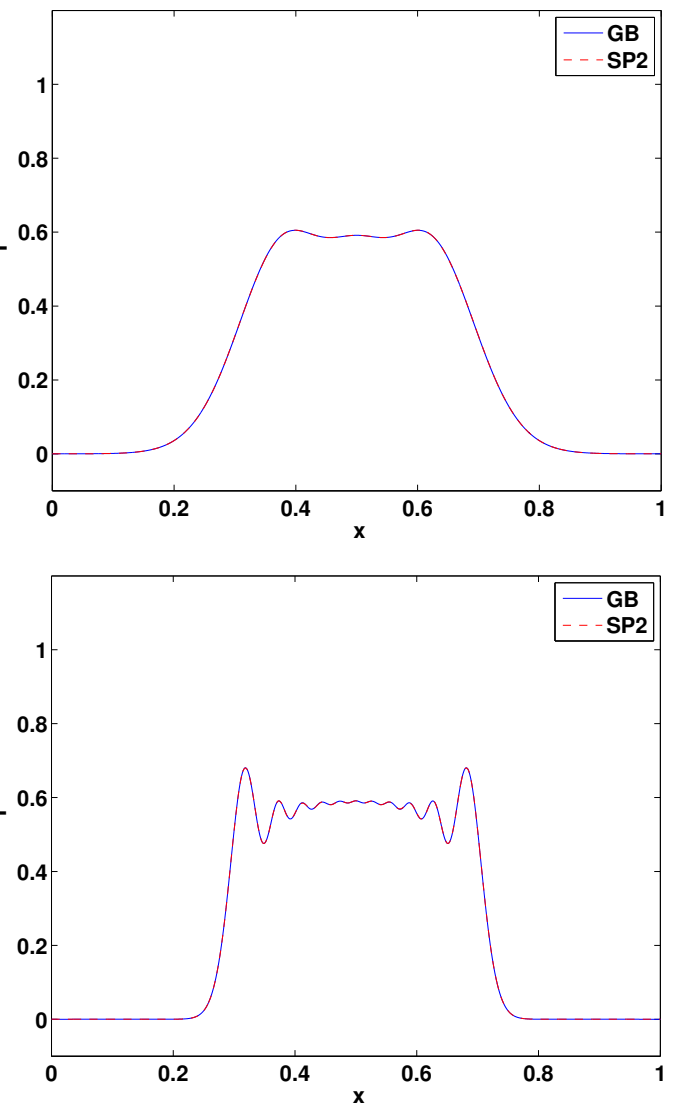

(c)

(e)

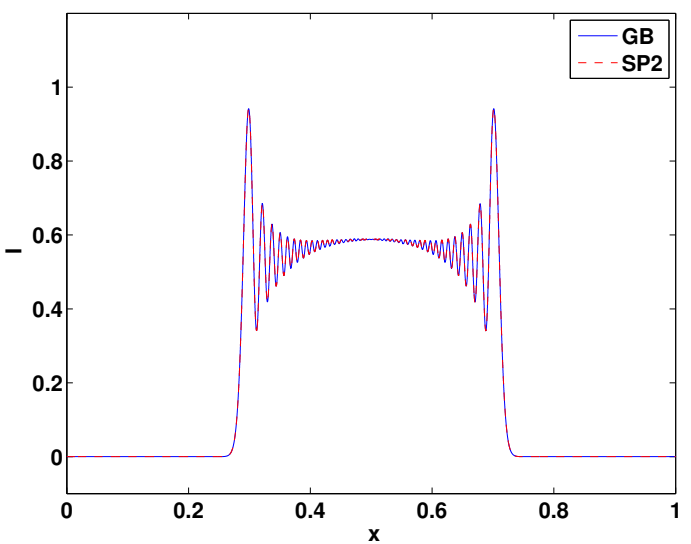

(b)

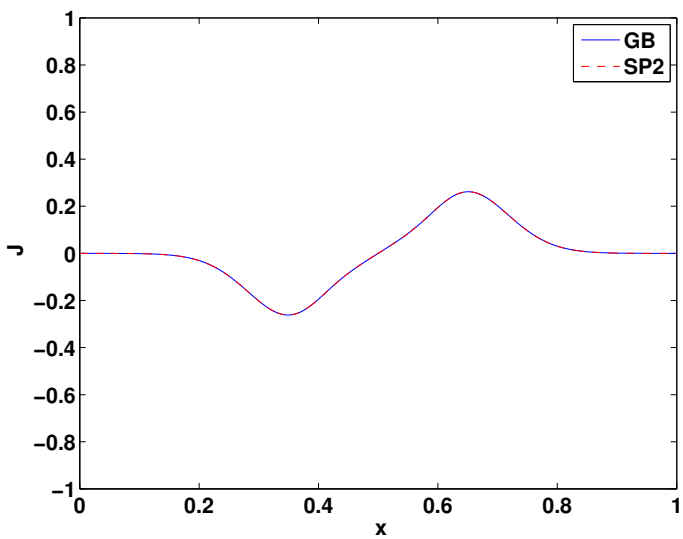

(d)
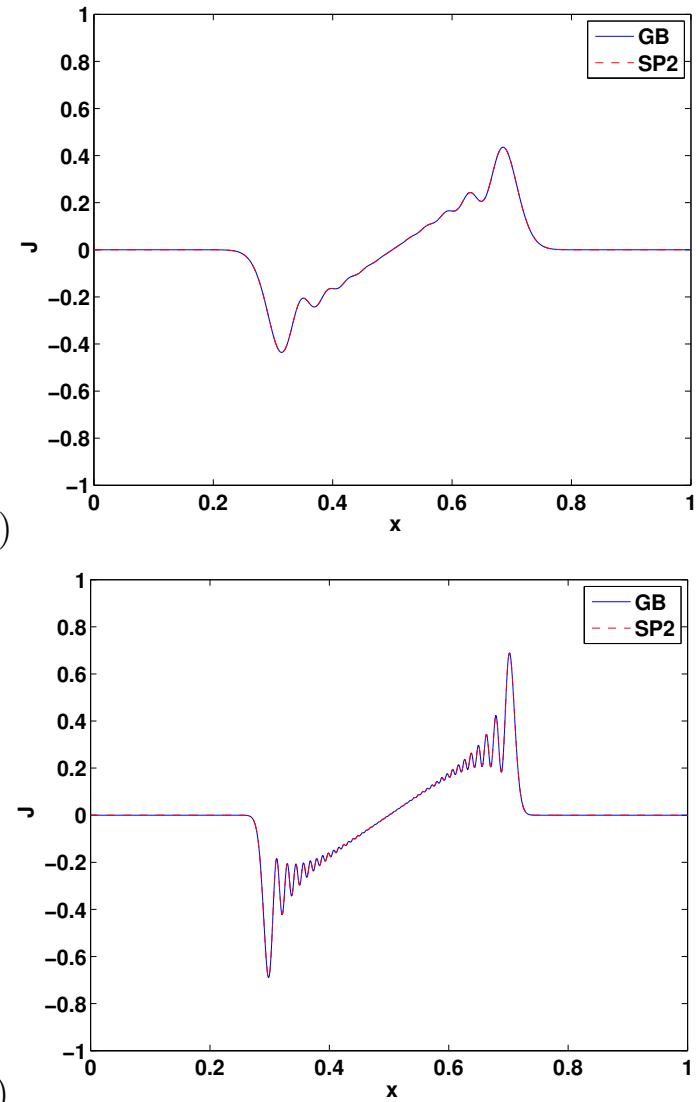

Figure 20: (Example 5.5) Comparison of the position densities computed by Eulerian Gaussian beams and the Strang Splitting Spectral scheme. The Eulerian Gaussian beam is based on the FBI transform for the initial condition with $\epsilon=1$. The Strang Splitting Spectral position density is computed with a mesh $N=2^{15}$ and $\Delta t=10^{-5}$. (a) The position densities with $\hbar=1 / 64$; (b) the current densities with $\hbar=1 / 64$; (c) the position densities with $\hbar=1 / 256$; (d) the current densities with $\hbar=1 / 256$; (e) the position densities with $\hbar=1 / 1024$; (f) the current densities with $\hbar=1 / 1024$. 
summation formula. This yields a very efficient method for computing semi-classical solutions to the Schrödinger equation. For instance, the proposed algorithm requires only $O\left(s N n^{2}\right)$ operations to compute $s$ different solutions with $s$ different initial wave functions under the influence of the same potential, where $N=O(1 / \hbar), \hbar$ is the Planck constant, and $n \ll N$ is the number of computed beams. Numerical experiments indicate that this Eulerian Gaussian beam approach yields accurate semi-classical solutions even at caustics.

\section{Acknowledgement}

Qian would like to thank Professors R. Burridge, S. Osher and J. Ralston for their interest and encouragement in this work. Qian is in part supported by NSF DMS-0810104 and in part by AFOSR grant \#FA9550-04-1-0143.

\section{References}

[1] V. M. Babich and V. S. Buldyrev. Asymptotic methods in short wave diffraction problems (in Russian). Nauka, Moscow, 1972.

[2] W. Bao, S. Jin, and A. Markowich. On time-splitting spectral approximations for the schrödinger equation in the semiclassical regime. J. Comput. Phys., 175:487-524, 2002.

[3] M.D. Buhmann. Radial Basis Functions. Cambridge University Press, 2003.

[4] R. Burridge and J. Qian. The fundamental solution of the time-dependent system of crystal optics. European J. Appl. Math., 17:63-94, 2006.

[5] J.C. Butcher. Implicit Runge-Kutta processes. Math. Comp., 18:50-64, 1964.

[6] V. Cerveny, M. Popov, and I. Psencik. Computation of wave fields in inhomogeneous mediaGaussian beam approach. Geophys. J. R. Astr. Soc., 70:109-128, 1982.

[7] L.-T. Cheng, H. Liu, and S. J. Osher. High frequency wave propagation in Schrodinger equations using the level set method. Comm. Math. Sci., 1:593-621, 2003.

[8] M. G. Crandall and P. L. Lions. Viscosity solutions of Hamilton-Jacobi equations. Trans. Amer. Math. Soc., 277:1-42, 1983.

[9] E. Hairer and M. Hairer. GniCodes - Matlab Programs for Geometric Numerical Integration. Springer, Berlin, 2002.

[10] E.J. Heller. Cellular dynamics: A new semiclassical approach to time-dependent quantum mechanics. J. Chem. Phys., 94:2723-2729, 1991.

[11] E.J. Heller. Guided Gaussian wave packets. Acc. Chem. Res., 39:127-134, 2006.

[12] N. R. Hill. Gaussian beam migration. Geophysics, 55:1416-1428, 1990. 
[13] L. Hörmander. On the existence and the regularity of solutions of linear pseudo-differential equations. L'Enseignement Mathematique, 17:99-163, 1971.

[14] S. Jin, H. Liu, S. Osher, and R. Tsai. Computing multivalued physical observables for the semi-classical limit of the Schrödinger equation. J. Comput. Phys., 205:222-241, 2005.

[15] S. Jin and S. J. Osher. A level set method for the computation of multivalued solutions to quasi-linear hyperbolic PDEs and Hamilton-Jacobi equations. Comm. Math. Sci., 1:575-591, 2003.

[16] E. Kluk, M.F. Herman, and H.L. Davis. Comparison of the propagation of semiclassical frozen gaussian wave functions with quantum propagation for a highly excited anharmonic oscillator. J. Chem. Phys., 84:326-334, 1986.

[17] S. Leung, J. Qian, and R. Burridge. Eulerian gaussian beams for high frequency wave propagation. Geophysics, 72:SM61-SM76, 2007.

[18] S. Leung, J. Qian, and S. Osher. A level set method for three-dimensional paraxial geometrical optics with multiple sources. Commun. Math. Sci., 2(4):643-672, 2004.

[19] D. Ludwig. Uniform asymptotic expansions at a caustic. Comm. Pure Appl. Math., XIX:215$250,1966$.

[20] P. Markowich, P. Pietra, C. Pohl, and H. Stimming. A Wigner-measure analysis of the dufortfrankel scheme for the schrödinger equation. SIAM J. Num. Anal., 40:1281-1310, 2002.

[21] P.A. Markowich, P. Pietra, and C. Pohl. Numerical approximation of quadratic observables of schrödinger-type equations in the semi-classical limit. Numer. Math., 81:595-630, 1999.

[22] A. Martinez. An Introduction to Semiclassical and Microlocal Analysis. Springer, 2002.

[23] V. P. Maslov. The Complex WKB Method for nonlinear equations. I Linear theory. Birkhauser Verlag, Basel, 1994.

[24] V. P. Maslov and M. V. Fedoriuk. Semi-classical approximation in quantum mechanics. D. Reidel Publishing Company, 1981.

[25] A. Messiah. Quantum Mechanics. John Wiley and Sons, New York, 1958.

[26] C. Min. Simplicial isosurfacing in arbitrary dimension and codimension. J. Comput. Phys., 190:295-310, 2003.

[27] S. Osher, L.-T. Cheng, M. Kang, H. Shim, and Y-H Tsai. Geometrical optics in a phase space based level set and Eulerian framework. J. Comput. Phys., 179:622-648, 2002.

[28] J. Qian, L.-T. Cheng, and S. J. Osher. A level set based Eulerian approach for anisotropic wave propagations. Wave Motion, 37:365-379, 2003.

[29] J. Qian and S. Leung. A level set method for paraxial multivalued traveltimes. J. Comput. Phys., 197:711-736, 2004. 
[30] J. Qian and S. Leung. A local level set method for paraxial multivalued geometric optics. SIAM J. Sci. Comput., 28:206-223, 2006.

[31] S. Qiu, F. Zhou, and P.E. Crandall. Discrete Gabor transforms with complexity O(NlogN). Singal Processing, 77:159-170, 1999.

[32] J. Ralston. Gaussian beams and the propagation of singularities. Studies in partial differential equations, 23:206-248, 1983.

[33] A. Shlivinski, E. Heyman, A. Boag, and C. Letrou. A phase-space beam summation formulation for ultrawide-band radiation. IEEE Trans. Antennas Propagat., 52:2042-2056, 2004.

[34] C. W. Shu and S. J. Osher. Efficient implementation of essentially non-oscillatory shock capturing schemes. J. Comput. Phys., 77:439-471, 1988.

[35] N. Tanushev. Superpositions and higher order Gaussian beams. Preprint, 2008.

[36] N. Tanushev, J. Qian, and J. Ralston. Mountain waves and Gaussian beams. SIAM J. Multi. Model. E Simul., 6:688-709, 2007.

[37] C.G. Vd Boogaart and R. Lienhart. Fast Gabor transformation for processing high quality audio. ICASSP 2006 Proceedings, 3:161-164, 2006. 